\title{
ROOK THEORY, COMPOSITIONS, AND ZETA FUNCTIONS
}

\author{
JAMES HAGLUND
}

\begin{abstract}
A new family of Dirichlet series having interesting combinatorial properties is introduced. Although they have no functional equation or Euler product, under the Riemann Hypothesis it is shown that these functions have no zeros in $\operatorname{Re}(s)>1 / 2$. Some identities in the ring of formal power series involving rook theory and continued fractions are developed.
\end{abstract}

\section{InTRODUCTION}

One of the most mysterious functions in analysis and number theory is the Riemann Zeta function:

$$
\zeta(s)=\prod_{i=1}^{\infty} \frac{1}{1-q_{i}^{-s}}, \quad \operatorname{Re}(s)>1,
$$

where $q_{1}=2, q_{2}=3$, is the sequence of prime numbers. In his great paper of 1859 , Riemann conjectured that $\zeta(s) \neq 0$ for $\operatorname{Re}(s)>1 / 2$. This is now known as the Riemann hypothesis ( $\mathrm{RH}$ for short) and is perhaps the greatest unsolved problem in mathematics.

In the author's dissertation [Ha1], the coefficient of $n^{-s}$ in $(\zeta(s)-1)^{k}$ was shown to have an interpretation in terms of combinatorial rook theory. More recent work [Ha2] has led to the investigation of a set of Dirichlet series with peculiar "pseudo Euler products". The simplest of these is

$$
\zeta_{2}(s)=\prod_{i=1}^{\infty} \frac{1}{1-\frac{q_{i}^{-s}}{1-\frac{q_{i+1}^{-s}}{1-\frac{q_{i+2}^{-s}}{1-\ldots}}}}, \quad \operatorname{Re}(s)>1 .
$$

Hoping to gain insight into the $\mathrm{RH}$ led the author to investigate the location of the zeros of the corresponding Dirichlet series, with the following result:

Theorem 1.1. a) $\zeta_{2}(s)$ can be analytically continued to the line $\operatorname{Re}(s)=0$.

b) If $\zeta_{2}(s)=0$, then $\zeta(k s)=0$ for some $k \in \mathbb{Z}^{+}$. Thus under the $R H \zeta_{2}(s)$ has no zeros in $\operatorname{Re}(s)>1 / 2$.

Received by the editors January 20, 1995 and, in revised form, November 6, 1995.

1991 Mathematics Subject Classification. Primary 11M41, 05A15.

Key words and phrases. Riemann zeta function, Riemann hypothesis, continued fraction, composition, rook theory, Euler product.

(C)1996 American Mathematical Society 
Although $\zeta_{2}(s)$ doesn't appear to satisfy a functional equation, combinatorially it has many similarities to $\zeta(s)$. For example,

$$
\zeta_{2}(s)^{-1}=\sum_{n=1}^{\infty} \frac{\mu_{2}(n)}{n^{s}},
$$

where

$$
\mu_{2}(n)= \begin{cases}0 & \text { if } q_{i}^{2} \mid n \text { or } q_{i} q_{i+1} \mid n \text { for some } i \\ (-1)^{\omega(n)} & \text { else, }\end{cases}
$$

with $\omega(n)$ the number of prime factors of $n$. This bears close resemblance to the well-known identity $\zeta(s)^{-1}=\sum_{n} \mu(n) n^{-s}$, where

$$
\mu(n)= \begin{cases}0 & \text { if } q_{i}^{2} \mid n \text { for some } i \\ (-1)^{\omega(n)} & \text { else. }\end{cases}
$$

One of the equivalent forms of the $\mathrm{RH}$ is that the function $M(x)$ (defined as $\left.\sum_{n \leq x} \mu(n)\right)$, is $O\left(x^{1 / 2+\epsilon}\right) \forall \epsilon>0$ [Tic, p.370]. We show this implies the same estimate holds for $\sum_{n \leq x} \mu_{2}(n)$ :

Theorem 1.2. Under the $R H$,

$$
\sum_{n \leq x} \mu_{2}(n)=O\left(x^{1 / 2+\epsilon}\right) \forall \epsilon>0 .
$$

Some unanswered questions are whether or not the assumption $\sum_{n \leq x} \mu_{2}(n)=$ $O\left(x^{1 / 2+\epsilon}\right) \forall \epsilon>0$ implies the $R H$, and if the line $\operatorname{Re}(s)=0$ forms a natural boundary for $\zeta_{2}(s)$.

Actually Theorems 1.1 and 1.2 have much more general versions, that hold for any of our class of Dirichlet series (which arise from more complicated continued fraction structures known as branching continued fractions, and which have an interpretation in terms of rook theory).

Remark on notation. $q_{i}$ will always denote the $i^{\text {th }}$ largest prime, but many of the theorems in this paper also hold true if we replace $q_{i}$ by some arbitrary permutation of the primes. So $p_{i}$ will denote the $i^{t h}$ member of some ordering of the primes $p_{1}, p_{2}, \ldots$, where each prime occurs once and only once in this list. Throughout the article $\sigma$ denotes $\operatorname{Re}(s)$. LHS and RHS are abbreviations for left hand side and right hand side, respectively.

\section{Combinatorial Properties}

In this section we define the functions $\zeta_{r}(s)$ and list the combinatorial identities which they satisfy, putting off the proofs until section 6 . For $r \geq 1$, set

$$
\zeta_{r}(s)=\sum_{n=1}^{\infty} \frac{1}{n^{s}} \prod_{i \geq 1}\left(\begin{array}{c}
\nu_{i}+\nu_{i-1}+\ldots+\nu_{i-r+1} \\
\nu_{i}
\end{array}\right), \quad n=\prod_{i} p_{i}^{\nu_{i}} .
$$

For example,

$$
\zeta_{2}(s)=\sum_{n=1}^{\infty} \frac{1}{n^{s}} \prod_{i \geq 1}\left(\begin{array}{c}
\nu_{i}+\nu_{i-1} \\
\nu_{i}
\end{array}\right)
$$


Note that $\zeta_{1}(s)=\zeta(s)$, and that different orderings of the primes $p_{1}, p_{2}, \ldots$ yield different Dirichlet series for $r>1$ but not for $r=1$. If $s=\sigma+i$,

$$
\begin{aligned}
\left|\zeta_{r}(s)\right| & \leq \zeta_{r}(\sigma) \leq \sum_{n=1}^{\infty} \frac{1}{n^{\sigma}} \prod_{i \geq 1}\left(\begin{array}{c}
\nu_{i}+\nu_{i-1}+\ldots+\nu_{1} \\
\nu_{i}
\end{array}\right) \\
& =\sum_{n=1}^{\infty} \frac{1}{n^{\sigma}}\left(\begin{array}{c}
\sum_{i} \nu_{i} \\
\nu_{1}, \nu_{2}, \ldots
\end{array}\right)=\frac{1}{1-\sum_{p} p^{-\sigma}}
\end{aligned}
$$

provided $\sigma>\beta$, where $\beta$ is the real solution to the equation $\sum_{p} p^{-\beta}=1$. Note that $\beta<2$. Thus $\zeta_{r}(s)$ converges for $\sigma>\beta_{r}$, for some $\beta_{r}$ satisfying $1 \leq \beta_{r} \leq \beta$ (section 3 contains more on $\beta_{r}$ ). All of the combinatorial properties of $\zeta_{r}(s)$ listed in this section hold for sure if $\sigma>\beta$. Some, such as the product identity for $\zeta_{r}$ below, hold for $\sigma>1$.

Pseudo Euler product. As mentioned earlier, $\zeta_{r}(\mathrm{~s})$ has a factorization, into generalized continued fractions, for $r>2$ as well. For example,

$$
\zeta_{3}(s)=\prod_{i=1}^{\infty} \frac{1}{1-\frac{p_{i}^{-s}}{\left(1-\frac{p_{i+1}^{-s}}{\left(1-\frac{p_{i+2}^{-s}}{1-\ldots}\right)\left(1-\frac{p_{i+3}^{-s}}{1-\ldots}\right)}\right)\left(1-\frac{p_{i+2}^{-s}}{\left(1-\frac{p_{i+3}^{-s}}{1-\ldots}\right)\left(1-\frac{p_{i+4}^{-s}}{1-\ldots}\right)}\right)}} .
$$

Here each term branches into 2 new terms, and in general $\zeta_{r}(s)$ has a product where each term branches into $r-1$ new terms

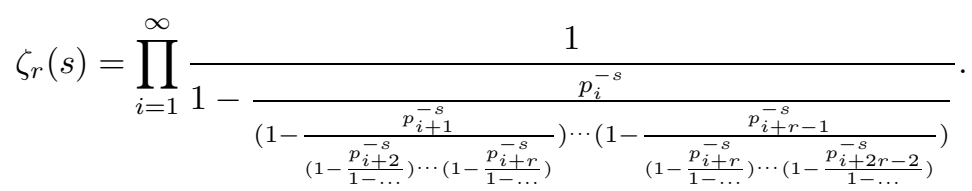

It is easy to describe the coefficient of $n^{-s}$ in $\zeta_{r}(s)^{-1}$. Define $\mu_{r}(n)$ as follows:

$$
\mu_{r}(n)= \begin{cases}0 & \text { if } p_{i} p_{j} \mid n \text { for some } i, j \text { with }|i-j| \leq r-1 \\ (-1)^{\omega(n)} & \text { else. }\end{cases}
$$

For example, if $p_{1}=2, p_{2}=3$, et cetera, then $\mu_{2}(21)=1$, but $\mu_{3}(21)=0$ since $p_{2} p_{4} \mid 21$. Note that $\mu_{1}(n)=\mu(n)$.

\section{Theorem 2.1.}

$$
\zeta_{r}(s)^{-1}=\sum_{n=1}^{\infty} \frac{\mu_{r}(n)}{n^{s}} .
$$

Corollary 2.2. $\zeta_{r}(s)$ has an analytic continuation to the line $\sigma=1$, and is zero free for $\sigma>1$.

Proof of the Corollary. Since $\sum_{n} \mu_{r}(n) n^{-s}$ converges absolutely and hence uniformly on compact subsets of $\sigma>1$, it represents an analytic function throughout that region. This function agrees with $\zeta_{r}(s)^{-1}$ for $\sigma>\beta$, so by uniqueness of analytic continuation also agrees with $\zeta_{r}(s)^{-1}$ for $\sigma>1$. Since $\left|\zeta_{r}(s)^{-1}\right|<\infty$ in this region, $\zeta_{r}(s) \neq 0$ for $\sigma>1$. 
Some other combinatorial properties of interest:

$$
\zeta_{r}(s)^{x}=\sum_{n=1}^{\infty} \frac{1}{n^{s}} \prod_{i \geq 1}\left(\begin{array}{c}
\nu_{i}+\nu_{i-1}+\ldots+\nu_{i-r+1}+x-1 \\
\nu_{i}
\end{array}\right), \quad x \in \mathbb{C}, n=\prod_{i} p_{i}^{\nu_{i}}
$$

and

$$
\zeta_{r}(s)=\exp \left(\sum_{n=2}^{\infty} \frac{1}{n^{s}} \frac{1}{\nu_{b}} \prod_{i \geq b+1}\left(\begin{array}{c}
\nu_{i}+\nu_{i-1}+\ldots+\nu_{i-r+1}-1 \\
\nu_{i}
\end{array}\right)\right), \quad n=\prod_{i} p_{i}^{\nu_{i}},
$$

where $b=b(n)=\min _{\nu_{i}>0} i$. The first identity holds as long as $\zeta_{r}(\sigma)<2$.

\section{Domain of Convergence}

In section 2 we mentioned that the series representation for $\zeta_{r}(s)$ converges for $\sigma>\beta$. In this section we show $\zeta_{2}(s)$ converges for $\sigma>1$, assuming we are dealing with the canonical ordering of primes $q_{1}=2, q_{2}=3$, et cetera. Using the trivial estimate $\left(\begin{array}{l}n \\ k\end{array}\right) \leq 2^{n}$, we have

$$
\begin{gathered}
\zeta_{2}(\sigma)=\sum_{n=1}^{\infty} \frac{1}{n^{\sigma}} \prod_{i \geq 1}\left(\begin{array}{c}
\nu_{i}+\nu_{i-1} \\
\nu_{i}
\end{array}\right) \\
=\sum_{a, b, c, d, e, \ldots} \frac{\left(\begin{array}{c}
a+b \\
b
\end{array}\right)\left(\begin{array}{c}
b+c \\
c
\end{array}\right)\left(\begin{array}{c}
c+d \\
d
\end{array}\right)\left(\begin{array}{c}
d+e \\
e
\end{array}\right)\left(\begin{array}{c}
e+f \\
f
\end{array}\right) \ldots}{2^{a \sigma} 3^{b \sigma} 5^{c \sigma} 7^{d \sigma} 11^{e \sigma} 13^{f \sigma} \ldots} \\
<\sum_{a, b, c, d, e, \ldots} \frac{\left(\begin{array}{c}
a+b \\
b
\end{array}\right)\left(\begin{array}{c}
b+c \\
c
\end{array}\right)\left(\begin{array}{c}
c+d \\
d
\end{array}\right) 2^{d+e} 2^{e+f} 2^{f+g} \ldots}{2^{a \sigma} 3^{b \sigma} 5^{c \sigma} 7^{d \sigma} 11^{e \sigma} 13^{f \sigma} \ldots} \\
\leq \sum_{a, b, c, d} \frac{\left(\begin{array}{c}
a+b \\
b
\end{array}\right)\left(\begin{array}{c}
b+c \\
c
\end{array}\right)\left(\begin{array}{c}
c+d \\
d
\end{array}\right) 2^{d}}{2^{a \sigma} 3^{b \sigma} 5^{c \sigma} 7^{d \sigma}} \prod_{p \geq 11} \frac{1}{1-4 / p^{\sigma}} .
\end{gathered}
$$

The sum on the right can be evaluated by setting $r=2, x=-1, x_{1}=-1 / 2^{\sigma}, x_{2}=$ $-1 / 3^{\sigma}, x_{3}=-1 / 5^{\sigma}, x_{4}=-2 / 7^{\sigma}$, and $x_{j}=0$ for $j>4$ in Theorem 6.2 (proved in section 6 ). The result is that for $\sigma>\beta$,

$$
\begin{gathered}
\sum_{a, b, c, d} \frac{\left(\begin{array}{c}
a+b \\
b
\end{array}\right)\left(\begin{array}{c}
b+c \\
c
\end{array}\right)\left(\begin{array}{c}
c+d \\
d
\end{array}\right) 2^{d}}{2^{a \sigma} 3^{b \sigma} 5^{c \sigma} 7^{d \sigma}} \\
=\frac{1}{1-\left(\frac{1}{2^{\sigma}}+\frac{1}{3^{\sigma}}+\frac{1}{5^{\sigma}}+\frac{2}{7^{\sigma}}-\frac{1}{10^{\sigma}}-\frac{2}{14^{\sigma}}-\frac{2}{21^{\sigma}}\right)} .
\end{gathered}
$$

If the LHS of this equation were to approach a pole, the denominator of the RHS would have to approach zero. Graphing it on a computer, we find that the denominator is positive for $\sigma>1$ (and first becomes zero around $\sigma=.97$ ), and so the 
LHS converges for $\sigma>1$. Since

$$
\zeta_{2}(\sigma) \leq \sum_{a, b, c, d} \frac{\left(\begin{array}{c}
a+b \\
b
\end{array}\right)\left(\begin{array}{c}
b+c \\
c
\end{array}\right)\left(\begin{array}{c}
c+d \\
d
\end{array}\right) 2^{d}}{2^{a \sigma} 3^{b \sigma} 5^{c \sigma} 7^{d \sigma}} \prod_{p \geq 11} \frac{1}{1-4 / p^{\sigma}},
$$

and since the product on the right converges for $\sigma>1$, we see that the domain of convergence for $\zeta_{2}(s)$ is $\sigma>1$.

For $r>2, \zeta_{r}(\sigma)$ does not converge for all $\sigma>1$ since

$$
\zeta_{r}(\sigma)<\sum_{a, b, c} \frac{\left(\begin{array}{c}
a+b \\
b
\end{array}\right)\left(\begin{array}{c}
a+b+c \\
c
\end{array}\right)}{2^{a \sigma} 3^{b \sigma} 5^{c \sigma}}=\frac{1}{1-\left(1 / 2^{\sigma}+1 / 3^{\sigma}+1 / 5^{\sigma}\right)}
$$

which has a pole in $\sigma>1$ since $1 / 2+1 / 3+1 / 5>1$. For some values of $r$, a better upper bound than $\beta$ for the domain of convergence of $\zeta_{r}(s)$ can be obtained by extending the argument given above for $\zeta_{2}(s)$.

\section{Analytic Continuation}

Theorem 4.1. Set

$$
C_{i}(s)=\frac{1}{1-\frac{p_{i}^{-s}}{1-\frac{p_{i+1}^{-s}}{1-\frac{p_{i+2}^{-s}}{1-\ldots}}}}, \quad \sigma>1 .
$$

Then $C_{i}(s)$ can be analytically continued to the line $\sigma=0$.

Proof. First a lemma:

Lemma 4.2. For any $m, N \in \mathbb{Z}^{+}$

$$
\sum_{\substack{n \\ \nu(n)=m \\ b(n)=N}} D_{2}(n) \leq 8^{m}
$$

where

$$
D_{2}(n)=\frac{1}{\nu_{b}} \prod_{j=b+1}^{\infty}\left(\begin{array}{c}
\nu_{j}+\nu_{j-1}-1 \\
\nu_{j}
\end{array}\right),
$$

$\nu(n)=\sum_{i} \nu_{i}$, and $b=b(n)=\min _{\nu_{i}>0} i$ as before.

Proof. Note that for any $k>N$, if $\nu_{k}>0$ and $\nu_{k-1}=0$ then $\left(\begin{array}{c}\nu_{j}+\nu_{j-1}-1 \\ \nu_{j}\end{array}\right)=0$ and so $D_{2}(n)=0$. Thus we need only consider integers of the form $p_{N}^{\nu_{N}} p_{N+1}^{\nu_{N+1}} \cdots p_{k}^{\nu_{k}}$, where $\nu_{j}>0$ for $N \leq j \leq k$, with $k$ in the range $N \leq k \leq N+m-1$. We have

$$
\begin{gathered}
\sum_{\substack{n \\
\nu(n)=m \\
b(n)=N}} \frac{1}{\nu_{N}} \prod_{j=N+1}^{\infty}\left(\begin{array}{c}
\nu_{j}+\nu_{j-1}-1 \\
\nu_{j}
\end{array}\right) \leq \sum_{\substack{n \\
\nu(n)=m, b(n)=N \\
\nu_{j}>0, N \leq j \leq k}} \frac{1}{\nu_{N}} \prod_{j=N+1}^{\infty} 2^{\nu_{j}+\nu_{j-1}} \\
\leq 4^{m} 2^{m}=8^{m},
\end{gathered}
$$

since $2^{m}$ is an upper bound for the number of solutions to $\nu_{N}+\nu_{N+1}+\ldots+\nu_{k}=$ $m, \nu_{j}>0$ for $N \leq j \leq k$. 
Continuing with the proof of Theorem 4.1, fix $\delta>0$, and choose $N$ big enough so that $j \geq N$ implies $p_{j}^{\delta}>9$ and also $N \geq i$. By Corollary 6.6, for $\sigma$ sufficiently large,

$$
C_{N}(s)=\exp \left(\sum_{\substack{n \\ b(n)=N}} \frac{1}{n^{s}} D_{2}(n)\right) .
$$

By Lemma 4.2,

$$
\begin{gathered}
\left|\sum_{b(n)=N} \frac{1}{n^{s}} D_{2}(n)\right| \leq \sum_{\substack { m=1 \\
\begin{subarray}{c}{n \\
b(n)=N \\
\nu(n)=m{ m = 1 \\
\begin{subarray} { c } { n \\
b ( n ) = N \\
\nu ( n ) = m } }\end{subarray}} \frac{1}{9^{m}} D_{2}(n) \\
\leq \sum_{m=1}^{\infty} \frac{8^{m}}{9^{m}}<\infty, \quad \sigma>\delta .
\end{gathered}
$$

Hence $C_{N}(s)$ is the exponential of a bounded function in $\sigma>\delta$. Since $C_{i}(s)$ can be expressed as a rational function in $C_{N}(s)$-for example,

$$
C_{1}(s)=\frac{1}{1-C_{2}(s) p_{1}^{-s}}
$$

-we see that $C_{i}(s)$ is a meromorphic function in $\sigma>\delta$ for any $\delta>0$ and thus for $\sigma>0$.

Remark. This proof can easily be modified to show that the branching continued fractions occurring in the product formula for $\zeta_{r}(\mathrm{~s})$ can also be continued to the line $\sigma=0$. It is not clear whether they can be extended beyond $\sigma=0$.

Theorem 4.3. Assume that $C_{i}(s)=\exp (g(s))$, where $g(\mathrm{~s})$ is bounded in $\sigma>\delta$. Then $\prod_{j=1}^{i} C_{j}(s)$ is zero free in $\sigma>\delta$.

Proof. If $i=1$, the product in question is the exponential of a bounded function and is thus zero free. So assume $i>1$. As is well-known, the finite continued fraction

$$
\frac{1}{1-\frac{x_{1}}{1-\frac{x_{2}}{\cdots 1-\frac{x_{n-1}}{1-x_{n}}}}}
$$

can be written as

$$
\frac{E_{2}\left(-x_{2},-x_{3}, \ldots,-x_{n}\right)}{E_{2}\left(-x_{1},-x_{2}, \ldots,-x_{n}\right)}
$$


where $E_{2}$ is a certain polynomial (the exact definition of $E_{2}$ follows Theorem 6.1). Note that for $j \leq i-1, C_{j}(s)$ can be written as the finite continued fraction

$$
\frac{1}{1-\frac{p_{j}^{-s}}{1-\frac{p_{j+1}^{-s}}{\cdots 1-\frac{C_{i}(s)}{p_{i-1}^{s}}}}} .
$$

Hence

$$
\begin{aligned}
\prod_{j=1}^{i} C_{j}= & C_{i}(s) \prod_{j=1}^{i-1} \frac{E_{2}\left(-p_{j+1}^{-s},-p_{j+2}^{-s}, \ldots,-C_{i}(s) p_{i-1}^{-s}\right)}{E_{2}\left(-p_{j}^{-s},-p_{j+1}^{-s}, \ldots,-C_{i}(s) p_{i-1}^{-s}\right)} \\
& =\frac{C_{i}(s)}{E_{2}\left(-p_{1}^{-s},-p_{2}^{-s}, \ldots,-C_{i}(s) p_{i-1}^{-s}\right)} .
\end{aligned}
$$

Since $C_{i}(s)$ is zero free and bounded for $\sigma>\delta$, the theorem follows.

Call a permutation $p_{1}, p_{2}, \ldots$ of the primes bounded if $p_{j}=q_{i}$ implies $|j-i| \leq B$ for some constant $B$ and all $i, j \geq 1$.

Theorem 4.4. Let $p_{1}, p_{2}, \ldots$ be a bounded permutation of the primes. Then, given $\delta>0$

$$
\zeta_{2}(s)=\exp (g(s)) \prod_{i=1}^{N} C_{i}(s) \prod_{k=1}^{M} \zeta(k s)^{a_{k}}
$$

for some integers $N, M$ which approach $\infty$ as $\delta \rightarrow 0$. The numbers $a_{k}$ are positive integers and $g(\mathrm{~s})$ is bounded in $\sigma>\delta\left(N, M\right.$, and $g$ depend on $\delta$ but the $a_{k}$ do not).

Before proving this we list some consequences of Theorems 4.1, 4.3, and 4.4.

Corollary 4.5. By the analytic continuation of $\zeta(s), \zeta_{2}(s)$ extends to a meromorphic function in $\sigma>0$; if $\zeta_{2}(s)=0$, then $\zeta(k s)=0$ for some $k \in \mathbb{Z}^{+}$.

Corollary 4.6. $\zeta_{2}(s)$ has a pole at $s=1 / k, k \in \mathbb{Z}^{+}$.

Since $s=0$ is a limit point of poles, it is an essential singularity of $\zeta_{2}(s)$. Similarly, any point on the line $\sigma=0$ is a limit point of zeros of the term

$$
\prod_{k=1}^{M} \zeta(k s)^{a_{k}}
$$

as $M \rightarrow \infty$, but some or all of these zeros could conceivably be cancelled by poles of $C_{i}(s)$ for some $i$, and the question of whether or not the line $\sigma=0$ is a natural boundary of $\zeta_{2}(s)$ remains open.

Proof of Theorem 4.4. The basic idea is to approximate $C_{i}(s)$ for large $i$ by

$$
\frac{1}{1-\frac{p_{i}^{-s}}{1-\frac{p_{i}^{-s}}{1-\frac{p_{i}^{-s}}{\cdots}}}},
$$


and show that

$$
\prod_{i>N_{0}} \frac{1}{1-\frac{p_{i}^{-s}}{1-\frac{p_{i}^{-s}}{\cdots}}}
$$

is approximately

$$
\prod_{i>N_{0}} \prod_{k=1}^{M} \frac{1}{\left(1-p_{i}^{-k s}\right)^{a_{k}}}
$$

which is a product of $\zeta$ functions modulo some bounded terms.

Fix $\delta>0$ and choose an integer $B \in \mathbb{Z}^{+}$such that $p_{j}=q_{i} \Longrightarrow|j-i| \leq B$. Next choose $N_{0}$ so that $N_{0} \geq B+1,\left(q_{N_{0}-B}\right)^{\delta}>8$, and for $j>N_{0}, C_{j}(s)=\exp (f(s))$ with $f(s)$ bounded in $\sigma>\delta$ (as we did in Theorem 4.1). For $\sigma$ sufficiently large,

$$
\zeta_{2}(s)=\prod_{i=1}^{N_{0}} C_{i}(s) \exp \left(\sum_{\substack{m, i \\ m \geq 1, i>N_{0}}} \frac{1}{m p_{i}^{m s}}+\sum_{\substack{\omega(n) \geq 2 \\ b(n)>N_{0}}} \frac{D_{2}(n)}{n^{s}}\right) .
$$

Choose $N_{1}>1 / \delta$. By Lemma 4.2 ,

$$
\left|\sum_{\omega(n) \geq 2} \sum_{\substack{n \\(n) \geq N_{1} \\ b(n)>N_{0}}} \frac{D_{2}(n)}{n^{s}}\right| \leq \sum_{k=N_{0}+1}^{\infty} \sum_{m=N_{1}}^{\infty} \frac{8^{m}}{\left(q_{k-B}\right)^{m \delta}}
$$

(since $b(n)=k \Longrightarrow$ all primes dividing $n$ are $\geq q_{k-B}$ )

$$
=\sum_{k=N_{0}+1}^{\infty}\left(\frac{8}{q_{k-B}^{\delta}}\right)^{N_{1}} \frac{1}{1-\frac{8}{q_{k-B}^{\delta}}}<\infty
$$

since $N_{1} \delta>1$ and $q_{N_{0}-B}^{\delta}>8$.

Next we consider the terms inside the exponential on the RHS of (1) satisfying $2 \leq \omega(n), 1<\nu(n)<N_{1}$. Let us start with $\nu(n)=2$. The term in question is

$$
\sum_{i>N_{0}} \frac{1}{p_{i}^{s} p_{i+1}^{s}}
$$

which is approximately $\sum_{i>N_{0}} 1 /\left(q_{i-B}\right)^{2 s}\left(\sum_{i} 1 / q_{i}^{s}\right.$ can be continued to the line $\sigma=$ 0 , where it has a natural boundary [Tic, p.215]). Thus

$$
\begin{gathered}
\left|\sum_{i>N_{0}} \frac{1}{\left(q_{i-B}\right)^{2 s}}-\frac{1}{p_{i}^{s} p_{i+1}^{s}}\right| \\
=\left|\sum_{i>N_{0}} \frac{1}{q_{i-B}^{2 s}}-\left(\frac{1}{q_{i-B}^{s}}+\frac{1}{p_{i+1}^{s}}-\frac{1}{q_{i-B}^{s}}\right)\left(\frac{1}{q_{i-B}^{s}}+\frac{1}{p_{i}^{s}}-\frac{1}{q_{i-B}^{s}}\right)\right| .
\end{gathered}
$$

By considering the real and imaginary parts of $q_{j}^{s}$, and using the mean value theorem of freshman calculus, $i>j$ implies

$$
\left|\frac{1}{q_{i}^{s}}-\frac{1}{q_{j}^{s}}\right| \leq \frac{\left(q_{i}-q_{j}\right) 4|s|}{q_{j}^{\sigma+1}} .
$$


Thus, multiplying out the RHS of (2),

$$
\begin{gathered}
\text { RHS of }(2)=O\left(\sum_{i>N_{0}} \frac{1}{q_{i-B}^{\sigma}}\left(\frac{q_{i+B+1}-q_{i-B}}{q_{i-B}^{1+\sigma}}\right)|s|\right) \\
+O\left(\sum_{i>N_{0}} \frac{\left(q_{i+B+1}-q_{i-B}\right)^{2}}{q_{i-B}^{2+2 \sigma}}|s|^{2}\right) .
\end{gathered}
$$

\section{Lemma 4.7.}

$$
\sum_{i} \frac{q_{i+k}-q_{i}}{q_{i}^{1+\sigma}}
$$

converges for $\sigma>0, k \geq 1$.

Proof. In a 1930's article, Cramér noted that

$$
\sum_{i} \frac{q_{i+1}-q_{i}}{q_{i} \log ^{a} q_{i}}
$$

converges for $a>1$, since the average value of $q_{i+1}-q_{i}$ is $\log q_{i}$ [Cra]. So assume $k>1$, and let $S_{x}=\Sigma_{q_{i} \leq x} q_{i+k}-q_{i}$. Basic facts about primes imply $S_{x}=O(x)(k$ fixed). Thus, by partial summation,

$$
\begin{gathered}
\sum_{q_{i} \leq x} \frac{q_{i+k}-q_{i}}{q_{i}^{1+\sigma}}=O\left(\frac{S_{x}}{x^{1+\sigma}}\right)+\sum_{q_{i} \leq x} O\left(q_{i}\right)\left(\frac{1}{q_{i}^{1+\sigma}}-\frac{1}{q_{i+1}^{1+\sigma}}\right) \\
=o(1)+\sum_{q_{i} \leq x} \frac{O\left(q_{i}\right)\left(q_{i+1}-q_{i}\right)}{q_{i}^{2+\sigma}}=O(1),
\end{gathered}
$$

using (3) and Cramér's result.

Continuing with the proof of 4.4, Lemma 4.7 implies the series on the RHS of (4) converge for $\sigma>0$. The same method can be used to deal with terms in (1) satisfying $\omega(n) \geq 2$ and $\nu(n)=m, 2<m<N_{1}$. For a fixed composition $\alpha$ of $m, \alpha_{0}+\alpha_{1}+\ldots+\alpha_{k}=m$, the difference between

$$
\sum_{i>N_{0}} \frac{1}{p_{i}^{\alpha_{0} s} p_{i+1}^{\alpha_{1} s} \ldots p_{i+k}^{\alpha_{k} s}} \frac{1}{\alpha_{0}} \prod_{j=1}^{k}\left(\begin{array}{c}
\alpha_{j}+\alpha_{j-1}-1 \\
\alpha_{j}
\end{array}\right)
$$

and

$$
\sum_{i>N_{0}} \frac{1}{q_{i-B}^{m s}} \frac{1}{\alpha_{0}} \prod_{j=1}^{k}\left(\begin{array}{c}
\alpha_{j}+\alpha_{j-1}-1 \\
\alpha_{j}
\end{array}\right)
$$

is bounded in modulus by a sum of terms of the form

$$
\sum_{i} O(1) \frac{\left(q_{i+a_{0}+B+1}-q_{i-B}\right)\left(q_{i+a_{1}+B+1}-q_{i-B}\right) \cdots\left(q_{i+a_{d}+B+1}-q_{i-B}\right)}{q_{i-B}^{m \sigma+d+1}},
$$


with $1 \leq a_{0}<a_{1}<\cdots<a_{d} \leq k$. Using Lemma 4.7 and elementary estimates, this also converges for $\sigma>0$. Putting everything together, for $\sigma$ sufficiently large we now have

$$
\zeta_{2}(s)=\prod_{i=1}^{N_{0}} C_{i}(s) \exp \left(\sum_{\substack{1 \leq m \\ N_{0}<i}} \frac{1}{m p_{i}^{m s}}+\sum_{\substack{2 \leq m<N_{1} \\ N_{0}<i}} \frac{d_{m}}{q_{i-B}^{m s}}+g_{1}(s)\right),
$$

where $g_{1}(s)$ is bounded in $\sigma>\delta$ and $d_{m}$ is a certain positive rational number. This can be rewritten as

$$
\zeta_{2}(s)=\prod_{i=1}^{N_{0}} C_{i}(s) \exp \left(\sum_{\substack{1 \leq m<N_{1} \\ N_{0}-B \leq i}} \frac{h(m)}{q_{i}^{m s}}+g_{2}(s)\right)
$$

with $h(m)$ also in $\mathbb{Q}^{+}$and $g_{2}$ bounded in $\sigma>\delta$. By construction,

$$
h(m)=\sum_{\substack{\alpha_{0}+\alpha_{1}+\ldots=m \\
\alpha_{i} \geq 1}} \frac{1}{\alpha_{0}} \prod_{j \geq 1}\left(\begin{array}{c}
\alpha_{j}+\alpha_{j-1}-1 \\
\alpha_{j}
\end{array}\right),
$$

and

$$
\frac{1}{1-\frac{x}{1-\frac{x}{1-\frac{x}{\cdots}}}}=\exp \left(\sum_{m=1}^{\infty} x^{m} h(m)\right)=\prod_{n \geq 1} \frac{1}{\left(1-x^{n}\right)^{a_{n}}}
$$

say. Eq. (8) implies $a_{n} \in \mathbb{Z}$, and by taking logarithms,

$$
a_{n}=\sum_{d \mid n} \frac{\mu(d)}{d} h(n / d)
$$

so $a_{1}=h(1)=1$ and $a_{2}=h(2)-h(1) / 2=1$.

Lemma 4.8. $2^{n-3} \leq a_{n} \leq 8^{n+1}$.

Proof. Eq. (9) implies

$$
h(n)-\sum_{\substack{d \mid n \\ d<n}} h(d) \leq a_{n} \leq h(n)+\sum_{\substack{d \mid n \\ d<n}} h(d) .
$$

Lemma 4.2 is equivalent to the statement $h(m) \leq 8^{m}$; this implies the upper bound on $a_{n}$ is $\leq 8^{n}+\ldots+8+1<8^{n+1}$. For the lower bound, given a composition $\alpha$ of $d, \alpha_{0}+\alpha_{1}+\ldots+\alpha_{k}=d$, where $d \mid n$ and $d \leq n / 2$, let $\beta(\alpha)$ be the composition $\alpha_{0}+\alpha_{1}+\ldots+\alpha_{k}+u+1+1+\ldots+1$ of $n$, where $u$ is the greatest integer in $(n+1) / 2$ and $\beta(\alpha)$ ends in $n-d-u$ ones. Clearly the product corresponding to $\beta(\alpha)$ in (7) is at least as big as the product corresponding to $\alpha$; hence 


$$
\begin{gathered}
a_{n} \geq \sum_{\begin{array}{c}
\alpha_{0}+\alpha_{1}+\ldots+\alpha_{j}=n \\
\text { a doesn't end in } u 1^{e} \\
\text { for some } e \geq 0
\end{array}} \frac{1}{\alpha_{0}} \prod_{i=1}^{j}\left(\begin{array}{c}
\alpha_{i}+\alpha_{i-1}-1 \\
\alpha_{i}
\end{array}\right) \\
\geq \sum_{\begin{array}{c}
\alpha_{0}+\alpha_{1}+\ldots+\alpha_{j}=n \\
\alpha \text { doesn't end in } u 1^{e} \\
\text { for some } e \geq 0 \text { and } \alpha_{0}=1
\end{array}} 1 \\
=\sum_{\alpha_{0}+\alpha_{1}+\ldots=n} 1-\sum_{\alpha_{0}=1}\left\{\begin{array}{c}
\# \text { of compositions of } n, \\
\alpha_{0}=1, \alpha \text { ends in } u 1^{e}
\end{array}\right\} \\
=2^{n-2}-\sum_{e=0}^{n-u-2} 2^{n-u-2-e}-1 \geq 2^{n-3} \text { for } n \geq 3 .
\end{gathered}
$$

By the argument below (1), if we replace $g_{2}(s)$ in (6) by

$$
g_{3}(s)+\sum_{\substack{N_{0}-B \leq i \\ N_{1} \leq m}} \frac{h(m)}{q_{i}^{m s}}
$$

then $g_{3}$ is also bounded in $\sigma>\delta$. We now have

$$
\zeta_{2}(s)=\exp \left(g_{3}(s)\right) \prod_{i=1}^{N_{0}} C_{i}(s) \prod_{i \geq N_{0}-B} \prod_{k=1}^{\infty} \frac{1}{\left(1-q_{i}^{-k s}\right)^{a_{k}}} .
$$

Since $a_{k} \in \mathbb{Z}^{+}$,

$$
\begin{aligned}
\prod_{i \geq N_{0}-B} \prod_{k=N_{1}+1}^{\infty} \frac{1}{\left(1-q_{i}^{-k s}\right)^{a_{k}}} \mid & \leq \exp \left(\sum_{\substack{N_{0}-B \leq i \\
N_{1} \leq m}} \frac{h(m)}{q_{i}^{m \sigma}}\right) \\
& =\exp \left(g_{4}(s)\right. \text { say, and } \\
\prod_{i=1}^{N_{0}-B} \prod_{k=1}^{N_{1}} \frac{1}{\left(1-q_{i}^{-k s}\right)^{a_{k}}} & =\exp \left(g_{5}(s)\right),
\end{aligned}
$$

where $g_{4}$ and $g_{5}$ are bounded in $\sigma>\delta$. Finally we get

$$
\zeta_{2}(s)=\exp (g(s)) \prod_{i=1}^{N_{0}} C_{i}(s) \prod_{i=1}^{\infty} \prod_{k=1}^{N_{1}} \frac{1}{\left(1-q_{i}^{-k s}\right)^{a_{k}}}
$$

for $\sigma$ sufficiently large, where $|g(s)| \leq g_{3}+g_{4}+g_{5}$ is bounded in $\sigma>\delta$. Theorem 4.4 now follows from the analytic continuation of the zeta function.

Remarks. The proof of Theorem 4.4 can easily be modified to show that given $\delta>0$, there exist integers $N_{r}, M_{r}$ such that $N_{r}, M_{r} \rightarrow \infty$ as $\delta \rightarrow 0$ and

$$
\zeta_{r}(s)=\exp (g(s, r)) \prod_{i=1}^{N_{r}} C_{i, r}(s) \prod_{k=1}^{M_{r}} \zeta(k s)^{a_{k}(r)}, \quad r>1,
$$

where $a_{k}(r) \in \mathbb{Z}^{+}, a_{1}(r) \equiv 1, a_{2}(r)=r-1$, and $2^{k-3} \leq a_{k}(r) \leq 2^{r k} r^{k}$ for $k \geq 3$. Here $C_{i, r}(s)$ is the $i^{\text {th }}$ branching continued fraction term in the product formula for $\zeta_{r}(s)$ (section 2), and $g(s, r)$ is bounded in $\sigma>\delta$. 
For "unbounded" permutations, the method of Theorem 4.4 can at least be used to continue $\zeta_{2}(s)$ to the line $\sigma=1 / 2$. For example, the series

$$
\sum_{i} \frac{1}{p_{i}^{s} p_{i+1}^{s}}
$$

is bounded in modulus by $2 \sum_{p} 1 / p^{2 \sigma}$, and terms where $\nu(n)>2$ also converge for $\sigma>1 / 2$. But the procedure breaks down when trying to continue $\zeta_{2}(s)$ beyond the half-line. Say $p_{2 i}=q_{i}$, and $p_{2 i+1}>10^{i}$; then the difference between

$$
\sum_{i} \frac{1}{p_{i}^{s} p_{i+1}^{s}} \text { and } \sum_{i} \frac{1}{q_{i}^{2 s}}
$$

only converges for $\sigma>1 / 2$ since

$$
\sum_{i} \frac{1}{p_{i}^{\sigma} p_{i+1}^{\sigma}}<2 \sum_{i} \frac{1}{10^{i \sigma}}<\infty \quad \text { for } \sigma>0 .
$$

5. The PaRTial Sums of $\mu_{r}(n)$

Recall that $M(x)=\Sigma_{n \leq x} \mu(n)$, and that the RH is equivalent to the condition $M(x)=O\left(x^{1 / 2+\epsilon}\right)$ for every $\epsilon>0$. The $\mathrm{RH}$ also implies a similar condition for $r>1$ :

Theorem 5.1. Assume the RH. Then

$$
\sum_{n \leq x} \mu_{r}(n)=O\left(x^{1 / 2+\epsilon}\right) \quad \forall \epsilon>0 .
$$

Proof. Theorem 5.1 is a special case of the following more general result:

Lemma 5.2. Start with an arbitrary ordering of the primes $p_{1}, p_{2}, \ldots$, and let $\mathcal{D}_{r}$ denote the following infinite set:

$$
\mathcal{D}_{r}=\left\{p_{i} p_{j} \mid 1 \leq i<j \leq i+r-1\right\} .
$$

Let $\mathcal{D}$ be any (possibly infinite) subset of $\mathcal{D}_{r}$. Then, assuming the $R H$,

$$
\sum_{d \mid n \stackrel{n \leq x}{\Longrightarrow} d \notin \mathcal{D}} \mu(n)=O\left(x^{1 / 2+\epsilon}\right) \text { for every } \epsilon>0 .
$$

Proof of Lemma 5.2. By inclusion-exclusion,

$$
\begin{aligned}
& \sum_{\substack{n \leq x \\
d \mid n \stackrel{n}{\Longrightarrow} d \notin \mathcal{D}}} \mu(n)=M(x)-\sum_{d \in \mathcal{D}} \mu(d) M(x / d, d)+\sum_{\substack{d_{1}, d_{2} \in \mathcal{D} \\
d_{1}<d_{2}}} \mu\left(\left[d_{1} d_{2}\right]\right) M\left(\frac{x}{\left[d_{1} d_{2}\right]},\left[d_{1} d_{2}\right]\right) \\
& -\sum_{\substack{d_{1}, d_{2}, d_{3} \in \mathcal{D} \\
d_{1}<d_{2}<d_{3}}} \mu\left(\left[d_{1} d_{2} d_{3}\right]\right) M\left(\frac{x}{\left[d_{1} d_{2} d_{3}\right]},\left[d_{1} d_{2} d_{3}\right]\right)+-+\ldots
\end{aligned}
$$

where $\left[d_{1} d_{2} \ldots d_{s}\right]=$ the least common multiple of $d_{1}, d_{2}, \ldots, d_{s}$ and

$$
M(x, d)=\sum_{\substack{n \leq x \\(n, d)=1}} \mu(n) .
$$


It turns out that the RH also implies $M(x, d)=O\left(x^{1 / 2+\epsilon}\right)$ for any $d \leq x$; first we show by induction that

$$
M(x, d)=\sum_{p|m \stackrel{m}{\Longrightarrow} p| d} M(x / m)
$$

Proof. The proof is actually an induction on ordered pairs $(j, x)$, where $j=\omega(d)$. Clearly we can assume $d$ is squarefree. For $j=0, M(x, 1)=M(x)$. The case $x=0$ reduces to $0=0$. For $j>1$, by inclusion-exclusion,

$$
\begin{aligned}
M(x, d) & =M(x)-\sum_{p \mid d} \mu(p) M(x / p, p)+\sum_{\substack{p_{i}, p_{j} \mid d \\
i<j}} \mu\left(p_{i} p_{j}\right) M\left(x / p_{i} p_{j}, p_{i} p_{j}\right)-+-\ldots \\
& =\sum_{q \mid d} M(x / q, q)=\sum_{q \mid d} \sum_{\substack{k \\
p|k \stackrel{k}{\Longrightarrow} p| q}} M(x / q k) \quad \text { (by induction) } \\
& =\sum_{p|k \stackrel{k}{\Longrightarrow} p| d} M(x / k) . \quad \square
\end{aligned}
$$

Next note that for any $y$,

$$
\begin{gathered}
|M(y, d)| \leq \sum_{p|k \stackrel{k}{\Longrightarrow} p| d}|M(y / k)| \\
=O\left(\sum_{p|k \stackrel{k}{\Longrightarrow} p| d}(y / k)^{1 / 2+\epsilon}\right) \quad \text { by the RH } \\
=O\left(y^{1 / 2+\epsilon} \sum_{p|k \stackrel{k}{\Longrightarrow} p| d} 1 / k^{1 / 2+\epsilon}\right)=O\left(y^{1 / 2+\epsilon} \prod_{p \mid d} \frac{1}{1-p^{-1 / 2-\epsilon}}\right) .
\end{gathered}
$$

In (10) we can assume $d \leq x$, in which case

$$
\prod_{p \mid d} \frac{1}{1-p^{-1 / 2-\epsilon}} \leq \prod_{i=1}^{N} \frac{1}{1-q_{i}^{-1 / 2-\epsilon}}
$$

where $q_{i}$ is the $i^{t h}$ largest prime, and $N$ is chosen so that $\prod_{i=1}^{N} q_{i} \leq x<\prod_{i=1}^{N+1} q_{i}$. Thus $\sum_{i=1}^{N} \log q_{i} \leq \log x$. Since $\sum_{i=1}^{N} \log q_{i}=O\left(q_{N}\right)$ by the Prime Number Theorem, $q_{N}=O(\log x)$ and thus

$$
\prod_{i=1}^{N} \frac{1}{1-q_{i}^{-1 / 2-\epsilon}}=O\left(\exp O\left(\sum_{p \leq \log x} 1 / p^{1 / 2+\epsilon}\right)\right)=O\left(\exp \left(O\left(\log ^{1 / 2} x\right)\right)\right) .
$$


Using this estimate in (11), we get $M(y, d)=O\left(y^{1 / 2+\epsilon} \exp \left(O\left(\log ^{1 / 2} x\right)\right)\right)=$ $O\left(y^{1 / 2+\epsilon} x^{\epsilon}\right)$ uniformly in $d$ for $d \leq x$. Plugging this into (10) results in

$$
\begin{aligned}
\left|\sum_{d \mid n \stackrel{n \leq x}{\Longrightarrow} d \notin \mathcal{D}} \mu(n)\right| \leq & |M(x)|+\sum_{d \in \mathcal{D}}|M(x / d, d)|+\sum_{\substack{d_{1}, d_{2} \in \mathcal{D} \\
d_{1}<d_{2}}}\left|M\left(\frac{x}{\left[d_{1} d_{2}\right]},\left[d_{1} d_{2}\right]\right)\right|+\ldots \\
= & O\left(x^{\epsilon}\right) O\left(x^{1 / 2+\epsilon}\right)+O\left(x^{\epsilon}\right) \sum_{d \in \mathcal{D}} O\left((x / d)^{1 / 2+\epsilon}\right) \\
& +O\left(x^{\epsilon}\right) \sum_{\substack{d_{1}, d_{2} \in \mathcal{D} \\
d_{1}<d_{2}}} O\left(\left(x /\left[d_{1} d_{2}\right]\right)^{1 / 2+\epsilon}\right)+\ldots
\end{aligned}
$$

(where the implied constants are uniformly bounded)

$$
=O\left(x^{1 / 2+2 \epsilon} \sum_{k \leq x} \frac{c_{k}}{k^{1 / 2+\epsilon}}\right)
$$

where $c_{k}$ is the number of ways that $k$ can be written in the form $\left[d_{1} d_{2} \cdots d_{j}\right], d_{i} \in \mathcal{D}$.

Given $k$ with $c_{k}>0$, we can uniquely decompose $k$ into squarefree "blocks" of primes $B_{1} B_{2} \ldots B_{m}$ where

a) all the primes occurring in block $B_{i}$ occur in the list $p_{1} p_{2} p_{3} \ldots$ before any of the primes in block $B_{i+1}$ for $1 \leq i \leq m-1$,

b) if $p_{j}$ divides $B_{i}$ and $p_{k}$ divides $B_{i+1}$ then $k-j \geq r$ (i.e. there are at least $r-1$ primes, not dividing $k$, between blocks $B_{i}$ and $B_{i+1}$ ), and

c) the number of blocks is maximal.

Example. If $k=p_{2} p_{4} p_{5} p_{8} p_{10} p_{13} p_{14}$, and $r=3$, then $B_{1}=p_{2} p_{4} p_{5}, B_{2}=p_{8} p_{10}$, and $B_{3}=p_{13} p_{14}$.

Now if $B_{j}$ consists of the product of an even number of primes, say $B_{j}=$ $p_{t_{1}} p_{t_{2}} \cdots p_{t_{2 q}}$, decompose $B_{j}$ into "mini-blocks" as follows:

$$
B_{j}=\left(p_{t_{1}} p_{t_{2}}\right)\left(p_{t_{3}} p_{t_{4}}\right) \cdots\left(p_{t_{2 q-1}} p_{t_{2 q}}\right) .
$$

If $B_{j}$ contains an odd number of primes, which must be greater than 1 if $c_{k}>0$, say $B_{j}=p_{t_{1}} \cdots p_{t_{2 q+1}}$, decompose $B_{j}$ into "mini-blocks" as follows:

$$
B_{j}=\left(p_{t_{1}} p_{t_{2}}\right) \cdots\left(p_{t_{2 q-3}} p_{t_{2 q-2}}\right)\left(p_{t_{2 q-1}} p_{t_{2 q}} p_{t_{2 q+1}}\right) .
$$

Hence

$$
\begin{aligned}
\sum_{\substack{k \leq x \\
c_{k}>0}} \frac{1}{k} \leq & \prod_{p_{i} \leq x}\left(1+\frac{1}{p_{i} p_{i+1}}+\frac{1}{p_{i} p_{i+2}}+\ldots+\frac{1}{p_{i} p_{i+r-1}}\right. \\
& \left.+\frac{1}{p_{i} p_{i+1} p_{i+2}}+\frac{1}{p_{i} p_{i+1} p_{i+3}}+\ldots+\frac{1}{p_{i} p_{i+r-1} p_{i+2 r-2}}\right)
\end{aligned}
$$

since the terms in the product on the right are the only possible mini-blocks in which $p_{i}$ is the prime with the smallest subscript.

Now a given prime $p_{i}$ occurs in terms in $\mathcal{D}$ of the form $p_{i} p_{i+j}$ or $p_{i-j} p_{i}$ for $1 \leq j \leq r-1$, so divides at most $2 r-2$ elements in $\mathcal{D}$. Thus there are at most $\omega(k)(2 r-2)$ elements in $\mathcal{D}$ which divide $k$. There are at most $2^{\omega(k)(2 r-2)}$ subsets 
TABLE I. The values of $M_{r}(x)$

$\begin{array}{rrrrrrrr}X & r & M_{r}(X) & \operatorname{Max} / x^{1 / 2} & X & r & M_{r}(X) & \operatorname{Max} / x^{1 / 2} \\ & & & & & & & \\ 10^{5} & 1 & -48 & 0.417 & 2 \times 10^{7} & 1 & -953 & 0.324 \\ & 2 & 307 & 1.236 & & 2 & -5958 & 1.343 \\ & 3 & 1349 & 4.320 & & 3 & -17030 & 3.818 \\ & 4 & 2724 & 8.620 & & 4 & -16134 & 3.616 \\ 10^{6} & 1 & 212 & 0.368 & 4 \times 10^{7} & 1 & -333 & 0.407 \\ & 2 & 178 & 0.510 & & 2 & -8954 & 1.507 \\ & 3 & 2002 & 2.167 & & 3 & -31911 & 5.090 \\ 10^{7} & 4 & 6623 & 6.679 & & 4 & -41805 & 6.636 \\ & 1 & 1037 & 0.361 & 6.5 \times 10^{7} & 1 & 1904 & 0.353 \\ & 2 & -2276 & 1.127 & & 2 & -10078 & 1.410 \\ & 3 & -7018 & 2.290 & & 3 & -45474 & 5.729 \\ & 4 & -1599 & 2.243 & & 4 & -69833 & 8.698\end{array}$

of these, which gives an upper bound for $c_{k}$. Hence

$$
\begin{aligned}
& \sum_{k \leq x} \frac{c_{k}}{k^{1 / 2+\epsilon}} \leq \prod_{p_{i} \leq x}\left(1+\frac{4^{2 r-2}}{\left(p_{i} p_{i+1}\right)^{1 / 2+\epsilon}}+\frac{4^{2 r-2}}{\left(p_{i} p_{i+2}\right)^{1 / 2+\epsilon}}+\ldots+\frac{4^{2 r-2}}{\left(p_{i} p_{i+r-1}\right)^{1 / 2+\epsilon}}\right. \\
& \left.\quad+\frac{8^{2 r-2}}{\left(p_{i} p_{i+1} p_{i+2}\right)^{1 / 2+\epsilon}}+\frac{8^{2 r-2}}{\left(p_{i} p_{i+1} p_{i+3}\right)^{1 / 2+\epsilon}}+\ldots+\frac{8^{2 r-2}}{\left(p_{i} p_{i+r-1} p_{i+2 r-2}\right)^{1 / 2+\epsilon}}\right) \\
& \leq \prod_{p_{i} \leq x}\left(1+\frac{4^{2 r-2}(r-1)}{\min \left(p_{i}, p_{i+1}, \ldots, p_{i+r-1}\right)^{1+2 \epsilon}}+\frac{8^{2 r-2}\left(\begin{array}{c}
2 r-2 \\
2
\end{array}\right)}{\min \left(p_{i}, p_{i+1}, \ldots, p_{i+2 r-2}\right)^{3 / 2+3 \epsilon}}\right)
\end{aligned}
$$

(since there are $\left(\begin{array}{c}2 r-2 \\ 2\end{array}\right)$ triples $p_{i} p_{j} p_{t}, 1 \leq i<j<t \leq i+2 r-2, i$ fixed)

$$
\leq \prod_{p_{i} \leq x}\left(1+\frac{4^{2 r-2}(r-1)}{p_{i}^{1+2 \epsilon}}+\frac{8^{2 r-2}\left(\begin{array}{c}
2 r-2 \\
2
\end{array}\right)}{p_{i}^{3 / 2+3 \epsilon}}\right)^{2 r-1}
$$

(note the exponent $2 r-1 ; p_{i}=\min \left(p_{a}, p_{a+1}, \ldots, p_{a+2 r-2}\right)$ for at most $2 r-1$ values of $a$ )

$$
\leq \prod_{p_{i} \leq x}\left(1+\frac{d_{r}}{p_{i}^{1+2 \epsilon}}\right)^{2 r-1} \leq A_{r} \prod_{2 d_{r}<p_{i} \leq x}\left(1+\frac{d_{r}}{p_{i}^{1+2 \epsilon}}\right)^{2 r-1}
$$

for some constants $d_{r}$ and $A_{r}$. Now

$$
\log \left(\prod_{2 d_{r}<p_{i} \leq x}\left(1+\frac{d_{r}}{p_{i}^{1+2 \epsilon}}\right)^{2 r-1}\right)=O\left(\sum_{2 d_{r}<p_{i} \leq x} \frac{d_{r}(2 r-1)}{p_{i}^{1+2 \epsilon}}\right)=O(1) .
$$

Thus

$$
\sum_{k \leq x} \frac{c_{k}}{k^{1 / 2+\epsilon}}=O(1)
$$

which together with (12) completes the proof of Lemma 5.2. 
In Table I, $M_{r}(x)=\sum_{n \leq x} \mu_{r}(n)$, and Max $/ x^{1 / 2}=\left(\max _{y \leq x}\left|M_{r}(y)\right|\right) /\left(x^{1 / 2}\right)$. The author would like to thank Charles Grassl of CRAY Research for running the program on a CRAY C-90 at Mendota Heights, Minnesota. The CPU time was 86 seconds.

\section{Some Identities in the Ring of Formal Power Series}

Several of the combinatorial identities involving $\zeta_{r}(s)$ have more general forms in the ring of formal power series, discovered while working on problems involving rook theory. An $n \times n$ chessboard is an $n \times n$ grid of squares; the lower left hand square has (column,row) coordinates $(1,1)$. A Ferrers board $B$ is a subset of squares of the chessboard with the property that if square $(i, j) \in B$ then all of the squares below it and to the right of square $(i, j)$ are also in $B$. A Ferrers board can be described in terms of its "column heights", $c_{1} \leq c_{2} \leq \ldots \leq c_{n}$, where $B$ consists of the lowest $c_{i}$ squares in the $i^{\text {th }}$ column, for $1 \leq i \leq n$. See Figure 1 . Note that the first several columns of this board have height 0 .

One of the most interesting theorems about Ferrers boards is due to Goldman, Joichi, and White, which says [GJW];

$$
\sum_{k=0}^{n} x(x-1) \cdots(x-k+1) r_{n-k}(B)=\prod_{i=1}^{n}\left(x+c_{i}-i+1\right), \quad x \in \mathbb{C},
$$

where $r_{j}(B)=$ the number of ways of placing $j$ non-attacking rooks (no two in the same row or column) on $B$. Using this, the author showed in his dissertation [Ha1] that rook placements are related to compositions of vectors. Given a vector $\mathbf{v} \in \mathbb{N}^{t}$, let $g_{k}(\mathbf{v})$ be the number of ways of writing $\mathbf{v}$ as the sum of $k$ nonzero vectors, each in $\mathbb{N}^{t}$, and with each coordinate equal to zero or one. These sums were originally studied by MacMahon; they are called unitary compositions ([Mac], p.158).

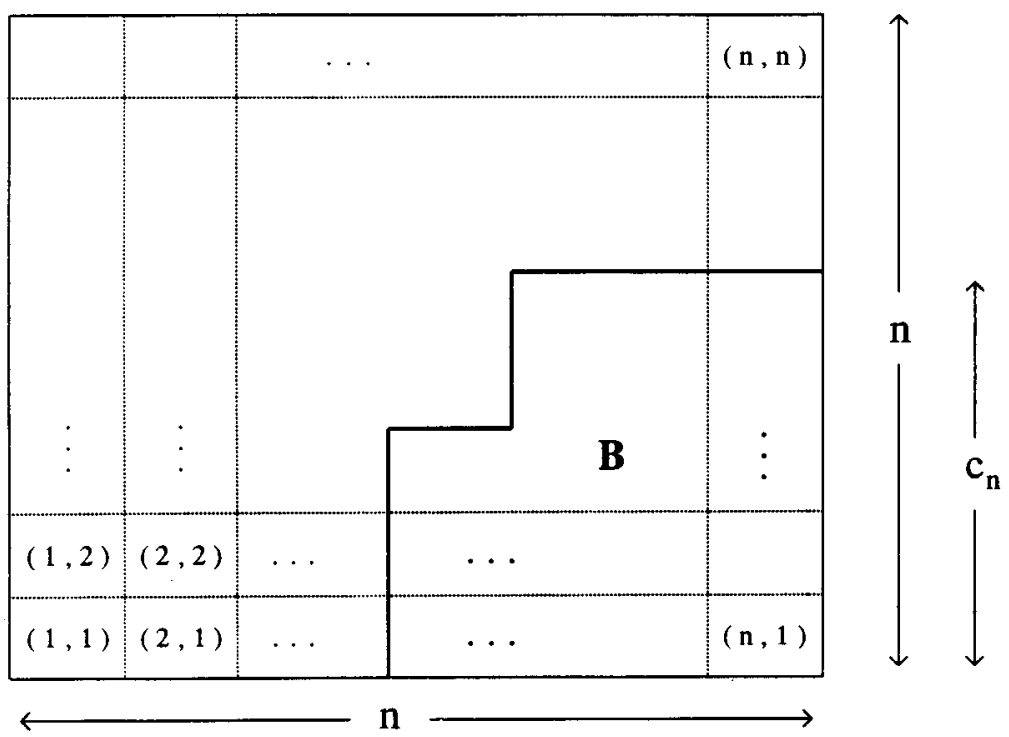

Figure 1. The Ferrers board $B$ 


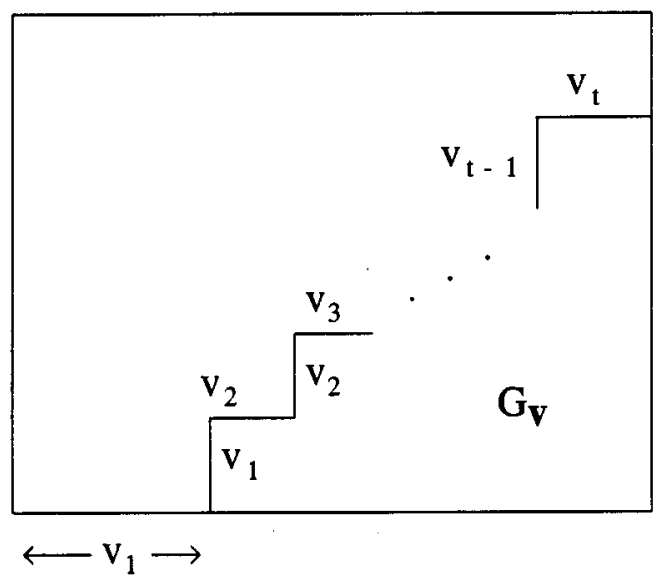

Figure 2. The Ferrers board $G_{\mathbf{v}}$

Example. Let $\mathbf{v}=(2,1)$. Then $g_{2}(\mathbf{v})=2$ since

$$
(2,1)=(1,1)+(1,0)=(1,0)+(1,1) .
$$

Theorem 3.2.6 of [Ha1] says that

$$
g_{k}(\mathbf{v})=\frac{k ! r_{n-k}\left(G_{\mathbf{v}}\right)}{\prod_{i} v_{i} !},
$$

where $n=\sum v_{i}$ and $G_{\mathbf{v}}$ is the Ferrers board in Figure 2 (the first $v_{1}$ columns have height zero).

The boards $G_{\mathbf{v}}$ are also connected to Simon Newcomb's problem (originally studied by MacMahon) which asks for the number of permutations of a multiset with a specified number of descents. A permutation $\sigma$ of a multiset $M$ is a linear list of the elements of $M: \sigma_{1} \sigma_{2} \cdots \sigma_{\# M}$. A descent of $\sigma$ is a value of $i$ such that $\sigma_{i}>\sigma_{i+1}$. Let $N_{k}(\mathbf{v})$ be the number of permutations of the multiset consisting of $v_{i}$ copies of $i, 1 \leq i \leq t$, with exactly $k-1$ descents. Riordan and Kaplansky showed that $[\mathrm{K}-\mathrm{R}]$

$$
N_{k}(\mathbf{v})=\frac{T_{k-1}\left(G_{\mathbf{v}}\right)}{\prod_{i} v_{i} !}
$$

where $T_{j}(B)$ equals the number of ways of placing $j$ non-attacking rooks on the $n \times n$ chessboard with exactly $j$ on the board $B$ (which is a subset of the $n \times n$ grid). For more recent work on compositions and Simon Newcomb's problem see [An1], [An2], [An3], [An4], [D-R], [F-S], [Ha2], [Si1], and [Si2].

There is a natural way of generalizing the Simon Newcomb problem which has been studied by Rawlings [Raw]. Define an $r$-descent of a permutation $\sigma$ to be a value of $i$ such that $\sigma_{i}>\sigma_{i+1}+r-1$. Let $N_{k}(\mathbf{v}, r)$ be the number of permutations (of the multiset consisting of $v_{i}$ copies of $i, 1 \leq i \leq t$ ) with exactly $k-1 r$-descents (actually Rawlings considered a $q$-version which depends on both $r$ and $q$ ). Another result from $[\mathrm{Ha} 1]$ is that

$$
N_{k}(\mathbf{v}, r)=\frac{T_{k-1}\left(G_{\mathbf{v}, r}\right)}{\prod_{i} v_{i} !}
$$




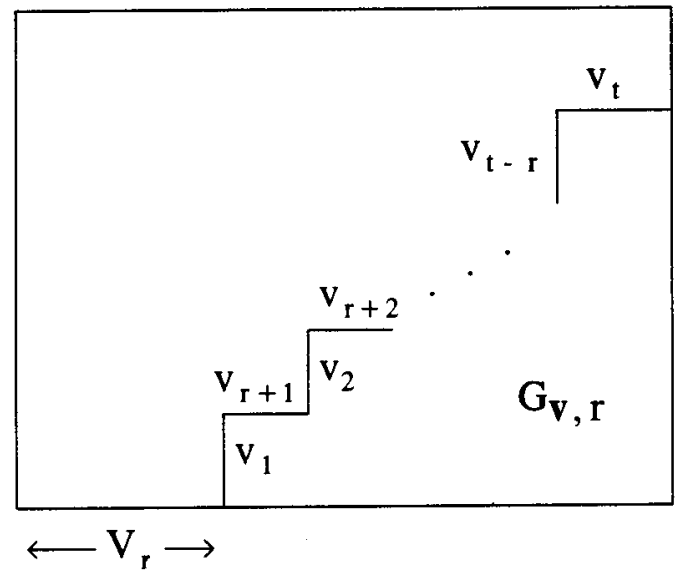

Figure 3 . The Ferrers board $G_{\mathbf{v}, r}$

where $G_{\mathbf{v}, r}$ is the Ferrers board of Figure 3 (here $V_{r}=v_{1}+v_{2}+\ldots+v_{r}$ ).

Can the rook numbers $r_{j}\left(G_{\mathbf{v}, r}\right)$ be interpreted in terms of compositions as in the $r=1$ case? Yes. Given a vector $\mathbf{v}=\left(v_{1}, v_{2}, \ldots, v_{t}\right)$, call $\mathbf{v} r$-unitary if

a) $v_{i}=0$ or 1 for all $i$, and

b) any two ones in $\mathbf{v}$ are separated by at least $r-1$ zeros (i.e. if $v_{i}=v_{j}=1$ and $i<j$, then $j-i \geq r)$.

For example, the vector $(1,0,1,0,0,1,0)$ is 2 -unitary but not 3 -unitary.

Let $g_{k}(\mathbf{v}, r)$ be the number of ways of writing $\mathbf{v}$ as the sum of $k$ nonzero $r$-unitary vectors. The following result is derived in [Ha2].

\section{Theorem 6.1.}

$$
g_{k}(\mathbf{v}, r)=\frac{k ! r_{n-k}\left(G_{\mathbf{v}, r}\right)}{\prod_{i} v_{i} !} .
$$

The definition of $g_{k}(\mathbf{v}, r)$ can be expressed in terms of generating functions as follows. Let $E_{r}\left(x_{1}, x_{2}, \ldots, x_{t}\right)$ denote the following function in the ring of formal power series:

$$
E_{r}\left(x_{1}, x_{2}, \ldots, x_{t}\right)=\sum_{\substack{\mathbf{v} \in \mathbb{N}^{t} \\ \mathbf{v} \text { is } r \text { unitary }}} \prod_{i} x_{i}^{v_{i}}
$$

and let

$$
E_{r}\left(x_{1}, x_{2}, \ldots\right)=\lim _{t \rightarrow \infty} E_{r}\left(x_{1}, x_{2}, \ldots, x_{t}\right) .
$$

Then by definition,

$$
\sum_{\mathbf{v} \in \mathbb{N}^{t}} \prod_{i} x_{i}^{v_{i}} g_{k}(\mathbf{v}, r)=\left(E_{r}\left(x_{1}, x_{2}, \ldots, x_{t}\right)-1\right)^{k} .
$$

In [Ha2], (15) and the next theorem are shown to follow from Theorem 6.1 and (13). A self-contained proof which doesn't rely on rook theory is included here. 
Theorem 6.2. In the ring of formal power series, for any $x \in \mathbb{C}$,

$$
E_{r}\left(x_{1}, x_{2}, \ldots\right)^{x}=\sum_{\mathbf{v}} \prod_{i} x_{i}^{v_{i}}\left(\begin{array}{c}
x-v_{i-1}-v_{i-2}-\ldots-v_{i-r+1} \\
v_{i}
\end{array}\right)
$$

(for $r=1$ this reduces to the binomial theorem since $E_{1}\left(x_{1}, x_{2}, \ldots\right)=\prod_{i}\left(1+x_{i}\right)$ ).

Proof. We start by showing that

$$
F_{r}\left(x_{1}, x_{2}, \ldots, x_{t}\right)^{x}=\sum_{v \in \mathbb{N}^{t}} \prod_{i} x_{i}^{v_{i}}\left(\begin{array}{c}
x-1+v_{i}+v_{i-1}+\ldots+v_{i-r+1} \\
v_{i}
\end{array}\right)
$$

where

$$
F_{r}\left(x_{1}, x_{2}, \ldots, x_{t}\right)=\sum_{\mathbf{v} \in \mathbb{N}^{t}} \prod_{i} x_{i}^{v_{i}}\left(\begin{array}{c}
v_{i}+v_{i-1}+\ldots+v_{i-r+1} \\
v_{i}
\end{array}\right) .
$$

First assume that $x \in \mathbb{N}$, and proceed by induction on $x$. For $x=1,(15)$ is trivial. For $x>1$,

$$
\begin{gathered}
F_{r}\left(x_{1}, x_{2}, \ldots, x_{t}\right)^{x}=F_{r}\left(x_{1}, x_{2}, \ldots, x_{t}\right)^{x-1} F_{r}\left(x_{1}, x_{2}, \ldots, x_{t}\right) \\
=\left(\sum_{\mathbf{v} \in \mathbb{N}^{t}} \prod_{i} x_{i}^{v_{i}}\left(\begin{array}{c}
x-2+v_{i}+v_{i-1}+\ldots+v_{i-r+1} \\
v_{i}
\end{array}\right)\right) \\
\times\left(\sum_{\mathbf{v} \in \mathbb{N}^{t}} \prod_{i} x_{i}^{v_{i}}\left(\begin{array}{c}
v_{i}+v_{i-1} \ldots+v_{i-r+1} \\
v_{i}
\end{array}\right)\right)
\end{gathered}
$$

(by the induction hypothesis)

$$
\begin{aligned}
=\sum_{\mathbf{v} \in \mathbb{N}^{t}} \prod_{i} x_{i}^{v_{i}} \sum_{\mathbf{0} \subseteq \mathbf{w} \subseteq \mathbf{v}} \prod_{i}\left(\begin{array}{c}
x-2+v_{i}-w_{i}+\ldots+v_{i-r+1}-w_{i-r+1} \\
v_{i}-w_{i}
\end{array}\right) \\
\times\left(\begin{array}{c}
w_{i}+\ldots+w_{i-r+1} \\
w_{i}
\end{array}\right) .
\end{aligned}
$$

Now we need to do a sub-induction on $t$. For $t=1$,

$$
\begin{aligned}
& \qquad \sum_{w_{1}=0}^{v_{1}}\left(\begin{array}{c}
x-2+v_{1}-w_{1} \\
v_{1}-w_{1}
\end{array}\right)\left(\begin{array}{l}
w_{1} \\
w_{1}
\end{array}\right) \\
& =\text { COEFFICIENT OF }\left(t^{v_{1}}\right) \text { IN } \frac{1}{(1-t)^{x-1}} \frac{1}{1-t}=\left(\begin{array}{c}
x-1+v_{1} \\
v_{1}
\end{array}\right) .
\end{aligned}
$$

For $t>1$, with $\mathbf{v}^{\prime}=\left(v_{1}, \ldots, v_{t-1}\right)$,

$$
\begin{gathered}
\sum_{\mathbf{0} \subseteq \mathbf{w} \subseteq \mathbf{v}} \prod_{i}\left(\begin{array}{c}
x-2+v_{i}-w_{i}+v_{i-1}-w_{i-1}+\ldots+v_{i-r+1}-w_{i-r+1} \\
v_{i}-w_{i}
\end{array}\right) \\
\times\left(\begin{array}{c}
w_{i}+\ldots+w_{i-r+1} \\
w_{i}
\end{array}\right) \\
=\sum_{0^{t-1} \subseteq \mathbf{w} \subseteq \mathbf{v}^{\prime}} \prod_{i=1}^{t-1}\left(\begin{array}{c}
x-2+v_{i}-w_{i}+\ldots+v_{i-r+1}-w_{i-r+1} \\
v_{i}-w_{i}
\end{array}\right)
\end{gathered}
$$




$$
\begin{aligned}
\times\left(\begin{array}{c}
w_{i}+\ldots+w_{i-r+1} \\
w_{i}
\end{array}\right) & \sum_{w_{t}=0}^{v_{t}}\left(\begin{array}{c}
x-2+v_{t}-w_{t}+\ldots+v_{t-r+1}-w_{t-r+1} \\
v_{t}-w_{t}
\end{array}\right) \\
& \times\left(\begin{array}{c}
w_{t}+\ldots+w_{t-r+1} \\
w_{t}
\end{array}\right) .
\end{aligned}
$$

The sum over $w_{t}$ is the COEFFICIENT OF $\left(t^{v_{t}}\right)$ IN

$$
\begin{aligned}
& \frac{1}{(1-t)^{x-1+v_{t-1}-w_{t-1}+\ldots+v_{t-r+1}-w_{t-r+1}}(1-t)^{w_{t-1}+\ldots+w_{t-r+1}+1}} \\
& =\text { COEFFICIENT OF }\left(t^{v_{t}}\right) \text { IN } \frac{1}{(1-t)^{x+v_{t-1}+\ldots+v_{t-r+1}}} \\
& =\left(\begin{array}{c}
x-1+v_{t}+\ldots+v_{t-r+1} \\
v_{t}
\end{array}\right) .
\end{aligned}
$$

Plugging this into (16) completes the induction on $t$ and $x$. Next we need to show $(15)$ is true for $x \in \mathbb{C}$. Setting $\left(F_{r}\left(x_{1}, x_{2}, \ldots, x_{t}\right)-1\right)^{k}=\sum_{\mathbf{v}} \prod_{i} x_{i}^{v_{i}} f_{k}(\mathbf{v}, r)$, we get

$$
\begin{gathered}
F_{r}\left(x_{1}, x_{2}, \ldots, x_{t}\right)^{x}=\left(1+F_{r}\left(x_{1}, x_{2}, \ldots, x_{t}\right)-1\right)^{x} \\
=\sum_{k=0}^{\infty}\left(\begin{array}{l}
x \\
k
\end{array}\right)\left(F_{r}\left(x_{1}, x_{2}, \ldots, x_{t}\right)-1\right)^{k} \\
=\sum_{\mathbf{v} \in \mathbb{N}^{t}} \prod_{i} x_{i}^{v_{i}} \sum_{k=0}^{\infty}\left(\begin{array}{l}
x \\
k
\end{array}\right) f_{k}(\mathbf{v}, r) \\
=\sum_{\mathbf{v} \in \mathbb{N}^{t}} \prod_{i} x_{i}^{v_{i}}\left(\begin{array}{c}
\left.x-1+v_{i}+v_{i-1}+\ldots+v_{i-r+1}\right) \\
v_{i}
\end{array}\right) \text { by (15). }
\end{gathered}
$$

Comparing the coefficient of $\prod_{i} x_{i}^{v_{i}}$ on both sides, we see that

$$
\sum_{k=0}^{\infty}\left(\begin{array}{l}
x \\
k
\end{array}\right) f_{k}(\mathbf{v}, r)=\prod_{i=1}^{t}\left(\begin{array}{c}
x-1+v_{i}+v_{i-1}+\ldots+v_{i-r+1} \\
v_{i}
\end{array}\right) .
$$

From the definition of $f_{k}(\mathbf{v}, r)$ given a few lines above, $f_{k}(\mathbf{v}, r)=0$ if $k>\sum_{i} v_{i}$. Both sides of (17) are thus polynomials in $x$ which agree if $x \in \mathbb{N}$ and hence are identically equal. To show that Theorem 6.2 follows, first let $x=-1$ in (15);

$$
F_{r}\left(x_{1}, x_{2}, \ldots, x_{t}\right)^{-1}=\sum_{\mathbf{v} \in \mathbb{N}^{t}} \prod_{i} x_{i}^{v_{i}}\left(\begin{array}{c}
-2+v_{i}+v_{i-1}+\ldots+v_{i-r+1} \\
v_{i}
\end{array}\right) .
$$

It is not hard to see that

$$
\prod_{i=1}^{t}\left(\begin{array}{cl}
-2+v_{i}+v_{i-1}+\ldots+v_{i-r+1} \\
v_{i}
\end{array}\right)= \begin{cases}0, & \text { if } \mathbf{v} \text { is not } r \text {-unitary } \\
(-1)^{v_{1}+\ldots+v_{t},}, & \text { if } \mathbf{v} \text { is } r \text {-unitary }\end{cases}
$$

which implies

$$
F_{r}\left(-x_{1},-x_{2}, \ldots,-x_{t}\right)^{-1}=E_{r}\left(x_{1}, x_{2}, \ldots, x_{t}\right),
$$


and letting $t \rightarrow \infty$ we get

$$
\begin{gathered}
E_{r}\left(x_{1}, x_{2}, \ldots\right)^{x}=F_{r}\left(-x_{1},-x_{2}, \ldots\right)^{-x} \\
=\sum_{\mathbf{v}} \prod_{i}\left(-x_{i}\right)^{v_{i}}\left(\begin{array}{c}
-x-1+v_{i}+v_{i-1}+\ldots+v_{i-r+1} \\
v_{i}
\end{array}\right) . \\
=\sum_{\mathbf{v}} \prod_{i} x_{i}^{v_{i}}\left(\begin{array}{c}
x-v_{i-1}-v_{i-2}-\ldots-v_{i-r+1} \\
v_{i}
\end{array}\right)
\end{gathered}
$$

since

$$
\left(\begin{array}{c}
m \\
k
\end{array}\right)=(-1)^{k}\left(\begin{array}{c}
-m+k-1 \\
k
\end{array}\right)
$$

Remark. The coefficients which occur in $F_{r}\left(x_{1}, x_{2}, \ldots\right)$ have a combinatorial interpretation:

$$
\prod_{i}\left(\begin{array}{c}
v_{i}+v_{i-1}+\ldots+v_{i-r+1} \\
v_{i}
\end{array}\right)
$$

is the number of permutations (of the multiset having $v_{i}$ copies of $i$ ) with no $r$ descents.

Continued fraction expansions. Let $C F_{r}\left(x_{1}, x_{2}, \ldots\right)$ denote the following branching continued fraction, where each term branches into $r-1$ new terms;

$$
\frac{1}{1+\frac{x_{1}}{\left(1+\frac{x_{2}}{\left(1+\frac{x_{3}}{(1+\ldots}\right) \cdots\left(1+\frac{x_{r}+1}{(1+\ldots}\right)}\right) \cdots\left(1+\frac{x_{r}}{\left(1+\frac{x_{r}+1}{(1+\ldots}\right) \cdots\left(1+\frac{x_{2}-1}{(1+\ldots}\right)}\right)}} .
$$

These structures have been the subject of several research articles and books, all of which are in Russian or Ukranian and difficult to obtain (see [RNT] Vol. 1a, pp.156-158). The rest of this section consists of several interesting combinatorial properties of these functions, which were derived independently by the author but most likely occur somewhere in the literature.

Theorem 6.3 (For $r=2$ this result appears in $[\mathrm{Per}])$. Let $C F_{r}\left(x_{1}, x_{2}, \ldots, x_{n}\right)$ denote the finite continued fraction $C F_{r}\left(x_{1}, x_{2}, \ldots, x_{n}, 0,0,0, \ldots\right)$. Then

$$
C F_{r}\left(x_{1}, x_{2}, \ldots, x_{n}\right)=\frac{E_{r}\left(x_{2}, x_{3}, \ldots, x_{n}\right)}{E_{r}\left(x_{1}, x_{2}, \ldots, x_{n}\right)} .
$$

Proof. By induction on $n$, both sides equaling $1 /\left(1+x_{1}\right)$ for $n=1$. For $n>1$,

$$
\begin{aligned}
C F_{r} & \left(x_{1}, x_{2}, \ldots, x_{n}\right) \\
& =\frac{1}{1+x_{1} C F_{r}\left(x_{2}, x_{3}, \ldots, x_{n}\right) C F_{r}\left(x_{3}, x_{4}, \ldots, x_{n}\right) \cdots C F_{r}\left(x_{r}, x_{r+1}, \ldots, x_{n}\right)} \\
& =\frac{1}{1+x_{1} \frac{E_{r}\left(x_{3}, x_{4}, \ldots, x_{n}\right)}{E_{r}\left(x_{2}, x_{3}, \ldots, x_{n}\right)} \cdots \frac{E_{r}\left(x_{r+1}, x_{r+2}, \ldots, x_{n}\right)}{E_{r}\left(x_{r}, x_{r+1}, \ldots, x_{n}\right)}} \quad \text { by induction } \\
& =\frac{E_{r}\left(x_{2}, \ldots, x_{n}\right)}{E_{r}\left(x_{2}, x_{3}, \ldots, x_{n}\right)+x_{1} E_{r}\left(x_{r+1}, x_{r+2}, \ldots, x_{n}\right)} \\
& =\frac{E_{r}\left(x_{2}, x_{3}, \ldots, x_{n}\right)}{E_{r}\left(x_{1}, x_{2}, \ldots, x_{n}\right)} .
\end{aligned}
$$




\section{Corollary 6.4.}

$$
F_{r}\left(x_{1}, x_{2}, \ldots\right)=\prod_{i=1}^{\infty} C F_{r}\left(-x_{i},-x_{i+1}, \ldots\right)
$$

provided that $E_{r}\left(\left|x_{1}\right|,\left|x_{2}\right|, \ldots\right)<2$.

Proof. By letting $n \rightarrow \infty$ in Theorem 6.3, the product on the right of Corollary 6.4 telescopes and so equals $E_{r}\left(-x_{1},-x_{2}, \ldots\right)^{-1}=F_{r}\left(x_{1}, x_{2}, \ldots\right)$. The condition $E_{r}\left(\left|x_{1}\right|,\left|x_{2}\right|, \ldots\right)<2$ guarantees that none of the terms in the product have zero for a denominator.

Theorem 6.5. (For $r=2$ this result occurs in some unpublished work of Doug Bowman) In the ring of formal power series,

$$
C F_{r}\left(-x_{1},-x_{2}, \ldots\right)^{x}=\sum_{\mathbf{v}} \prod_{i} x_{i}^{v_{i}}\left(\begin{array}{c}
x+v_{1}-1 \\
v_{1}
\end{array}\right) \prod_{i \geq 2}\left(\begin{array}{c}
v_{i}+\ldots+v_{i-r+1}-1 \\
v_{i}
\end{array}\right) .
$$

Proof. We have

$$
\begin{gathered}
C F_{r}\left(-x_{1},-x_{2}, \ldots,-x_{t}\right)^{x}=\frac{E_{r}\left(-x_{2},-x_{3}, \ldots-x_{t}\right)^{x}}{E_{r}\left(-x_{1},-x_{2}, \ldots,-x_{t}\right)^{x}} \quad \text { (Theorem 6.3) } \\
=\sum_{\substack{\mathbf{v} \in \mathbb{N}^{t} \\
v_{1}=0}} \prod_{i \geq 2}\left(-x_{i}\right)^{v_{i}}\left(\begin{array}{c}
x-v_{i-1}-\ldots-v_{i-r+1} \\
v_{i}
\end{array}\right) \\
\quad \times \sum_{\mathbf{v} \in \mathbb{N}^{t}} \prod_{i \geq 1}\left(-x_{i}\right)^{v_{i}}\left(\begin{array}{c}
-x-v_{i-1}-\ldots-v_{i-r+1} \\
v_{i}
\end{array}\right) \\
=\sum_{\mathbf{v} \in \mathbb{N}^{t}} \prod_{i}\left(-x_{i}\right)^{v_{i}}\left(\begin{array}{c}
-x \\
v_{1}
\end{array}\right) \sum_{w_{2}=0}^{v_{2}}\left(\begin{array}{c}
x \\
w_{2}
\end{array}\right)\left(\begin{array}{c}
-x-v_{1} \\
v_{2}-w_{2}
\end{array}\right) \\
\quad \times \sum_{w_{3}=0}^{v_{3}} \ldots \sum_{w_{t}=0}^{v_{t}}\left(\begin{array}{c}
x-w_{t-1} \ldots-w_{t-r+1} \\
w_{t}
\end{array}\right) \\
\times\left(\begin{array}{c}
-x-\left(v_{t-1}-w_{t-1}\right)-\ldots-\left(v_{t-r+1}-w_{t-r+1}\right) \\
v_{t}-w_{t}
\end{array}\right) .
\end{gathered}
$$

By the Vandermonde convolution, the sum over $w_{t}$ equals

$$
\left(\begin{array}{c}
-v_{t-1}-v_{t-2}-\ldots-v_{t-r+1} \\
v_{t}
\end{array}\right)
$$

and proceeding with an inductive argument we end up with

$$
\begin{gathered}
C F_{r}\left(-x_{1},-x_{2}, \ldots,-x_{t}\right)^{x}=\sum_{\mathbf{v} \in \mathbb{N}^{t}} \prod_{i}\left(-x_{i}\right)^{v_{i}}\left(\begin{array}{c}
-x \\
v_{1}
\end{array}\right) \prod_{i \geq 2}\left(\begin{array}{c}
-v_{i-1}-\ldots-v_{i-r+1} \\
v_{i}
\end{array}\right) \\
=\sum_{\mathbf{v} \in \mathbb{N}^{t}} \prod_{i} x_{i}^{v_{i}}\left(\begin{array}{c}
x+v_{1}-1 \\
v_{1}
\end{array}\right) \prod_{i \geq 2}\left(\begin{array}{c}
v_{i}+v_{i-1}+\ldots+v_{i-r+1}-1 \\
v_{i}
\end{array}\right)
\end{gathered}
$$

(since $\left.\left(\begin{array}{l}n \\ k\end{array}\right)=(-1)^{k}\left(\begin{array}{c}-n+k-1 \\ k\end{array}\right)\right)$. Letting $t \rightarrow \infty$ finishes the proof. 
Remark. A similar argument shows that

$$
\begin{gathered}
\prod_{i=1}^{m} C F_{r}\left(-x_{i},-x_{i+1}, \ldots\right)^{x}=\sum_{\mathbf{v}} \prod_{i} x_{i}^{v_{i}} \prod_{i=1}^{m}\left(\begin{array}{c}
x+v_{i}+\ldots+v_{i-r+1}-1 \\
v_{i}
\end{array}\right) \\
\times \prod_{i \geq m+1}\left(\begin{array}{c}
v_{i}+\ldots+v_{i-r+1}-1 \\
v_{i}
\end{array}\right) .
\end{gathered}
$$

Corollary 6.6. In the ring of formal power series,

$$
F_{r}\left(x_{1}, x_{2}, \ldots\right)=\exp \left(\sum_{\mathbf{v} \neq \mathbf{0}} \prod_{i} x_{i}^{v_{i}} \frac{1}{v_{b}} \prod_{i \geq b+1}\left(\begin{array}{c}
v_{i}+v_{i-1}+\ldots+v_{i-r+1}-1 \\
v_{i}
\end{array}\right),\right.
$$

where $b=b(\mathbf{v})=\min _{v_{i}>0} i$.

Proof. By Corollary 6.4,

$$
\begin{gathered}
\log F_{r}\left(x_{1}, x_{2}, \ldots\right)=\sum_{j=1}^{\infty} \log C F_{r}\left(-x_{j},-x_{j+1}, \ldots\right) \\
=\sum_{j=1}^{\infty} \log \left(1+C F_{r}\left(-x_{j}, \ldots\right)-1\right)=\sum_{j=1}^{\infty} \sum_{k=1}^{\infty} \frac{(-1)^{k+1}}{k}\left(C F_{r}\left(-x_{j}, \ldots\right)-1\right)^{k} \\
=\sum_{j=1}^{\infty} \sum_{k=1}^{\infty} \sum_{i=0}^{k}\left(\begin{array}{c}
k \\
i
\end{array}\right) \frac{(-1)^{k+1}}{k}\left(C F_{r}\left(-x_{j}, . .\right)\right)^{i}(-1)^{k-i} \\
=\sum_{j=1}^{\infty} \sum_{b(\mathbf{v})=j} \prod_{i} x_{i}^{v_{i}} \prod_{a \geq j+1}\left(\begin{array}{c}
v_{a}+\ldots+v_{a-r+1}-1 \\
v_{a}
\end{array}\right) \\
\quad \times \sum_{k=1}^{\infty} \sum_{i}\left(\begin{array}{c}
k \\
i
\end{array}\right) \frac{(-1)^{k+1+k-i}}{k}\left(\begin{array}{c}
i+v_{j}-1 \\
v_{j}
\end{array}\right)
\end{gathered}
$$

(by Theorem 6.5). Applying binomial inversion to the identity

$$
\sum_{k}\left(\begin{array}{l}
x \\
k
\end{array}\right)\left(\begin{array}{l}
n-1 \\
k-1
\end{array}\right)=\left(\begin{array}{c}
x+n-1 \\
n
\end{array}\right)
$$

the sum over $k$ and $i$ in (19) equals

$$
\begin{gathered}
\sum_{k \geq 1} \frac{(-1)^{k+1}}{k}\left(\begin{array}{c}
v_{j}-1 \\
k-1
\end{array}\right)=\int_{0}^{1} \sum_{k=1}^{v_{j}}(-1)^{k-1} x^{k-1}\left(\begin{array}{c}
v_{j}-1 \\
k-1
\end{array}\right) d x \\
=\int_{0}^{1}(1-x)^{v_{j}} d x=1 / v_{j}
\end{gathered}
$$

and the corollary follows. 
Remark. The coefficient

$$
\prod_{i \geq j+1}\left(\begin{array}{c}
v_{i}+v_{i-1}+\ldots+v_{i-r+1}-1 \\
v_{i}
\end{array}\right)
$$

also has a combinatorial interpretation; it is the number of permutations (of the multiset having $v_{i}$ copies of $i$ ) with no $r$-descents, and which end with $j$ (the smallest element).

Example 6.7. MacMahon noted that the Rogers-Ramanujan identities are equivalent to

$$
E_{2}\left(1, q, q^{2}, q^{3}, \ldots\right)=\frac{1}{1-q^{1}} \frac{1}{1-q^{4}} \frac{1}{1-q^{6}} \frac{1}{1-q^{9}} \frac{1}{1-q^{11}} \frac{1}{1-q^{14}} \ldots,
$$

and

$$
E_{2}\left(1, q^{2}, q^{3}, q^{4}, \ldots\right)=\frac{1}{1-q^{2}} \frac{1}{1-q^{3}} \frac{1}{1-q^{7}} \frac{1}{1-q^{8}} \frac{1}{1-q^{12}} \frac{1}{1-q^{13}} \ldots
$$

Taking the reciprocal of both sides of these equations, and using the combinatorial interpretation of the coefficients of $F_{2}\left(x_{1}, x_{2}, \ldots\right)$ (see the remark following the proof of Theorem 6.2), we get

$$
(1-q)\left(1-q^{4}\right)\left(1-q^{6}\right)\left(1-q^{9}\right) \cdots=\sum_{n \geq 0} q^{n} \sum_{\substack{\lambda \vdash n \\ \lambda_{i} \geq \lambda_{i+1}-1}}(-1)^{\# \text { parts }},
$$

and

$$
\left(1-q^{2}\right)\left(1-q^{3}\right)\left(1-q^{7}\right)\left(1-q^{8}\right) \cdots=\sum_{n \geq 0} q^{n} \sum_{\substack{\lambda \vdash n \\ \lambda_{i} \geq \lambda_{i+1}-1 \\ \lambda_{i} \geq 2}}(-1)^{\# \text { parts }} .
$$

Here the sums are over "semi-partitions" $\lambda$ of $n$ (we allow consecutive parts to increase, but only by at most one), and "\# parts" is the number of parts of $\lambda$.

\section{Some Other Dirichlet Series}

By definition, $\zeta_{r}(s)=F_{r}\left(1 / p_{1}^{s}, 1 / p_{2}^{s}, \ldots\right)$, and the combinatorial identities listed in section 2 are thus special cases of those in section 6 . Given an arbitrary subset $\mathcal{D}$ of $\mathcal{D}_{r}$, let

$$
\mu_{\mathcal{D}}(n)= \begin{cases}\mu(n), & \text { if } n \text { is not divisible by any } d \in \mathcal{D} ; \\ 0, & \text { else, }\end{cases}
$$

and set

$$
\zeta_{\mathcal{D}}(s)=\left(\sum_{n=1}^{\infty} \frac{\mu_{\mathcal{D}}(n)}{n^{s}}\right)^{-1}
$$

Then, by the same argument as in Corollary 2.2, Lemma 5.2 implies that (assuming the $\mathrm{RH}) \zeta_{\mathcal{D}}(s)$ has an analytic continuation to the line $\sigma=1 / 2$, and is zero free for $\sigma>1 / 2$. The choice $\mathcal{D}=\mathcal{D}_{r}$ is not the only choice for which $\zeta_{\mathcal{D}}(s)$ has a product representation; for example, if $\mathcal{D}=\left\{q_{2 i-1} q_{2 i} \mid i \geq 1\right\}$ then

$$
\zeta_{\mathcal{D}}(s)=\frac{1}{\left(1-2^{-s}-3^{-s}\right)} \frac{1}{\left(1-5^{-s}-7^{-s}\right)} \frac{1}{\left(1-11^{-s}-13^{-s}\right)} \ldots
$$


For another example, let $\mathcal{D}=\left\{p_{i} p_{i+1} \mid i \geq 1\right\} \cup\left\{p_{2 i} p_{2 i+2} \mid i \geq 1\right\}$. Then

$$
\zeta_{\mathcal{D}}(s)=\prod_{i \geq 1} \frac{1}{1 \text { odd }} 1-p_{i}^{-s}-\frac{p_{i+1}^{-s}}{1-p_{i+2}^{-s}-\frac{p_{i+3}^{-s}}{1-p_{i+4}^{-s}-\frac{p_{i+5}^{-s}}{1-\cdots}}}
$$

(continued fractions of this type involving powers of $q$ instead of primes have applications to partition theory; see [All]).

Extension to negative $r$. The coefficient of $1 / n^{s}$ in $\zeta_{r}(s)$ equals the number of permutations of the associated multiset having no $r$-descents. An $r$-descent is simply a value of $i$ such that $\sigma_{i}>\sigma_{i+1}+r-1$, and there is no problem in this definition if we allow $r<1$. For example, we should define

$$
\zeta_{0}(s)=\prod_{p}\left(1+\frac{1}{p^{s}}\right)
$$

since in order for a permutation to have no 0-descents, there must be an ascent of at least one at every spot, which is possible if and only if the multiset has distinct elements (i.e. $n$ is squarefree). Note that $\zeta_{0}(s)$ factors as $\zeta(s) / \zeta(2 s)$, which under the $\mathrm{RH}$ has all of its zeros on the line $\sigma=1 / 2$. In general define

$$
\zeta_{r}(s)=\sum_{n=1}^{\infty} \frac{\left|\mu_{1-r}(n)\right|}{n^{s}}, \quad r<1 .
$$

By (18) and Corollary 6.6 we can write

$$
\begin{gathered}
\zeta_{r}(s)=E_{1-r}\left(1 / p_{1}^{s}, 1 / p_{2}^{s}, \ldots\right) \quad(r<1) \\
=F_{1-r}\left(-1 / p_{1}^{s},-1 / p_{2}^{s}, \ldots\right)^{-1} \\
=\exp \left(\sum_{n>1} \frac{(-1)^{\nu(n)+1}}{n^{s} \nu_{b}} \prod_{j \geq b+1}\left(\begin{array}{c}
\nu_{j}+\nu_{j-1}+\ldots+\nu_{j-r+1}-1 \\
\nu_{j}
\end{array}\right)\right) .
\end{gathered}
$$

Using this identity the proof of Theorem 4.4 can be extended to show that, given $\delta>0$

$$
\zeta_{r}(s)=h_{r}(s) \prod_{k=1}^{N_{r}} \zeta(k s)^{a_{k}(r)}, \quad r<0,
$$

where $h_{r}(s)$ has no poles (but may have zeros) in $\sigma>\delta$ and $N_{r} \rightarrow \infty$ as $\delta \rightarrow 0$. The integers $a_{k}(r)$ are positive for $k$ odd and negative for $k$ even, which can be used to show that the line $\sigma=0$ forms a natural boundary for $\zeta_{r}(s), r<0$.

\section{8. $r$-Multiplicative Functions}

Although the coefficient of $n^{-s}$ in $\zeta_{r}(s)$,

$$
\prod_{i \geq 1}\left(\begin{array}{c}
\nu_{i}+\nu_{i-1}+\ldots+\nu_{i-r+1} \\
\nu_{i}
\end{array}\right)
$$


is not multiplicative, it does satisfy a certain generalized concept of multiplicativity. Call a function $h r$-multiplicative if $h(m n)=h(m) h(n)$ whenever $p_{i}\left|m, p_{j}\right| n \Longrightarrow$ $|j-i| \geq r$. The following properties are easily established:

Let $h$ and $x$ be two $r$-multiplicative functions. Then:

1) If $y(n)=\sum_{d \mid n} h(d) x(n / d)$, then $y$ is $r$-multiplicative.

2) If $\left(\sum_{n \geq 1} h(n) / n^{s}\right)^{-1}=\sum_{n \geq 1} w(n) / n^{s}$, then $w$ is $r$-multiplicative.

\section{Some Questions for Future Research}

1) Where are the poles of $\zeta_{r}(s)$ and what are their orders?

2) Does $\sum_{n \leq x} \mu_{r}(n)=O\left(x^{1 / 2+\epsilon}\right)$ for some $r>1$ imply $M(x)=O\left(x^{1 / 2+\epsilon}\right)$ ?

3) One of the interesting things about $\zeta_{2}(s)$ is that it admits a "natural" $q$ version. By replacing all binomial coefficients by $q$-binomial coefficients we have a function which equals $\zeta_{2}(s)$ for $q=1$ and $\zeta(s)$ for $q=0$. Where are the zeros of this function for $0<q<1$ ? (A different $q$-version of $\zeta(s)$, constructed by interpolating Carlitz's $q$-Bernoulli numbers, has been introduced by Satoh [Sat].)

4) Carlitz [Car] studied the polynomials $H_{n}(\lambda)$ defined by

$$
\frac{1-\lambda}{\zeta(s)-\lambda}=\sum_{n=1}^{\infty} \frac{H_{n}(\lambda)}{n^{s}}
$$

and Dillon and Roselle [D-R] noted the coefficients of $H_{n}(\lambda)$ are the Simon Newcomb numbers $N_{k}(\nu(n))$ described in section 6 . In the same paper Carlitz introduced generalized Bernoulli numbers $\beta(n)$ defined by

$$
\frac{\log \zeta(s)}{\zeta(s)-1}=\sum_{n=1}^{\infty} \frac{\beta(n)}{n^{s}}
$$

$\left(\beta\left(p_{1} p_{2} \cdots p_{k}\right)=B_{k}\right.$, the $k^{t h}$ Bernoulli number). If we replace $\zeta(s)$ by $\zeta_{r}(s)$ in (20) we generate the numbers $N_{k, r}(\nu(n))$ from section 6 . If we make the same substitution in (21), what properties will the corresponding numbers $\beta_{r}(n)$ have?

\section{ACKNOWLEDGEMENT}

The proof of the $r=2$ case of Theorem 5.1 was worked out in a conversation with Andrew Granville. The author would also like to thank the referees for bringing the literature involving branching continued fractions and various minor matters to the author's attention.

\section{REFERENCES}

[All] K. Alladi, Some New Observations on the Göllnitz-Gordon and Rogers-Ramanujan Identities, Trans. Amer. Math. Soc. 347 (1995), 897-914. MR 95h:11109

[An1] G. E. Andrews, The Theory of Partitions, Encyclopedia of Mathematics and its Applications, Vol. 2, Addison-Wesley, Reading, Mass., 1976. MR 58:27738

[An2] G. E. Andrews, The Theory of Compositions (I): The Ordered Factorizations of $n$ and a conjecture of C. Long, Canad. Math. Bull. 18 (1975), 479-484. MR 54:7363

[An3] G. E. Andrews, The Theory of Compositions (II): Simon Newcomb's Problem, Utilitas Math. 7 (1974), 33-54. MR 54:7364a

[An4] G. E. Andrews, The Theory of Compositions (III): the MacMahon Formula and the Stanton-Cowan Numbers, Utilitas Math. 9 (1976), 283-290. MR 54:7364b

[Car] L. Carlitz, Extended Bernoulli and Eulerian numbers, Duke Math. J. 31 (1964), 667-689. MR 29:5796

[Cra] H. Cramér, On the order of magnitude of the difference between consecutive prime numbers, Acta Arith. 2 (1936), 23-46. 
[D-R] J. F. Dillon and D. P. Roselle, Simon Newcomb's Problem, SIAM J. Appl. Math. 17 (1969), 1086-1093. MR 41:1553

[F-S] D. C. Foata and M. P. Schützenberger, On the rook polynomials of Ferrers relations, Colloq. Math. Soc. Janos Bolyai, 4, Combinatorial Theory and its Applications, vol. 2, North-Holland, Amsterdam, 1970, pp. (413-436). MR 50:12738

[Gra] A. Granville, Harald Cramér and the distribution of prime numbers, Scand. Actuar. J. 1995, 12-28. CMP 95:17

[GJW] J. Goldman, J. Joichi, and D. White, Rook theory I: Rook equivalence of Ferrers boards, Proc. Amer. Math. Soc. 52 (1975), 485-492. MR 55:2590

[H-W] G. H. Hardy and E. M. Wright, An Introduction to the Theory of Numbers, Fifth Edition, Clarendon Press, Oxford, 1979. MR 81i:10002

[Ha1] J. Haglund, Rook Placements, Compositions, and Permutations of Vectors, Doctoral Dissertation, University of Georgia, Athens, Georgia, 1993.

[Ha2] J. Haglund, Compositions and rook placements, preprint, 1994.

[Ha3] J. Haglund, Compositions and q-rook polynomials, in preparation.

[K-R] I. Kaplansky and J. Riordan, The problem of the rooks and its applications, Duke Math. J. 13 (1946), 259-268. MR 7:508d

[Mac] P. A. MacMahon, Combinatory Analysis, Vol. 1, Cambridge University Press, 1915.

[Per] O. Perron, Die Lehre von den Kettenbrüchen, Chelsea, New York, 1950. MR 12:254b

[Raw] D. Rawlings, The $(q-r)$ Simon Newcomb Problem, Linear and Multilinear Algebra 10 (1981), 253-260. MR 83k:05009

[Rio] J. Riordan, An Introduction to Combinatorial Analysis, John Wiley, New York, 1958. MR 20:3077

[RNT] Richard K. Guy (editor), Reviews in Number Theory 1973-83, Vol. 1a, Amer. Math. Soc., Providence, RI, 1984.

[Sat] J. Satoh, q-Analogue of Riemann's $\zeta$-function and $q$-Euler numbers, J. Number Theory 31 (1989), 346-362. MR 90d:11132

[Si1] R. Simion, On Compositions of Multisets, Doctoral Dissertation, The University of Pennsylvania, 1981.

[Si2] R. Simion, A multi-indexed Sturm sequence of polynomials and unimodality of certain combinatorial sequences, J. Combin. Theory Ser. A 36 (1984), 15-22. MR 85e:05015

[Sta] R. P. Stanley, Enumerative Combinatorics: Volume I, Wadsworth and Brooks/Cole, Pacific Grove, CA, 1986. MR 87j:05003

[Tic] E. C. Titchmarsh, The Theory of the Riemann Zeta-Function, Second Edition, Clarendon Press, 1986. MR 88c: 11049

Department of Mathematics, The University of Illinois at Urbana-Champaign, UrBANA, IL 61801

E-mail address: jhaglund@math.uiuc.edu 